Send Orders for Reprints to reprints@ benthamscience.ae

Journal Name, Year, Volume

1

\title{
Title: Elastin-like recombinamers as smart drug delivery systems
}

\author{
F. Javier Arias*a, Mercedes Santos ${ }^{\mathrm{a}}$, Arturo Ibáñez-Fonseca ${ }^{\mathrm{a}}, \mathrm{M}^{\mathrm{a}} \mathrm{Jesús}^{\text {Piñaa }}{ }^{\mathrm{a}}$ and Sofía Serrano ${ }^{\mathrm{a}}$
}

\author{
${ }^{a}$ BIOFORGE (Group for Advanced Materials and Nanobiotechnology), CIBER-BBN, University \\ Valladolid, 47011 Valladolid, Spain
}

\begin{abstract}
Drug delivery systems that are able to control the release of bioactive molecules and designed to carry drugs to target sites are of particular interest for tissue therapy. Moreover, systems comprising materials that can respond to environmental stimuli and promote self-assembly and higher order supramolecular organization are especially useful in the biomedical field. Suitable biomaterials for this purpose include elastin-like recombinamers (ELRs), a class of proteinaceous polymers bioinspired by natural elastin and designed using recombinant technologies. The self-assembly and thermoresponsive behaviour of these systems, along with their biodegradability, biocompatibility and well-defined composition as a result of their tailor-made design, make them particularly attractive for drug delivery.
\end{abstract}

\begin{abstract}
This review brings together information concerning ELR-based delivery systems that allow targeted delivery, especially ELR-drug recombinant fusion constructs, ELR-drug systems chemically bioconjugated in their monomeric and soluble forms, and drug encapsulation by nanoparticle-forming ELRs. Subsequently, we will focus on those drug carriers in which smart release is triggered by $\mathrm{pH}$ or temperature with a particular focus on cancer treatments in which both enhanced permeability retention (EPR) and local mild hyperthermia cause ELRs to become aggregated or form well-defined nanoparticles at the site of action. Systems for controlled drug release based on depots and hydrogels that act as both a support and reservoir in which drugs can be stored will be described, and their applications in drug delivery discussed. Finally, smart drug-delivery systems not based on ELRs, including those comprising proteins, synthetic polymers and non-polymeric systems, will also be briefly discussed.
\end{abstract}

Keywords: Elastin-like Recombinamers, Drug delivery, Smart systems, Self-Assembly, Hydrogels, Nanocarriers.

\section{INTRODUCTION}

The next generation of biomaterials will require new components capable of undergoing bioresponsive selfassembly and higher order supramolecular organization. In this sense, polymers that show lower critical solution temperature (LCST) phase-transition behaviour are a class of materials that can be used as fundamental pillars which are capable of interacting with biological systems at the molecular level [1].

A great variety of drug-delivery systems have been developed to obtain more efficient treatments and to deal with the largest possible number of diseases [2]. Recently, one of the main goals has been to develop systems that are capable of exhibiting a responsive behaviour to different environmental changes. Both polymeric and non-polymeric structures have been included in the wide variety of drugdelivery systems designed, including micelles, liposomes, polymer-drug conjugates or inorganic nanoparticles, each with different benefits $[3,4]$.

\footnotetext{
*Address correspondence to this author at BIOFORGE (Group for Advanced Materials and Nanobiotechnology), CIBER-BBN, University of Valladolid, Paseo de Belén 1947011 Valladolid, Spain; Tel: +34983423394; E-mail: arias@bioforge.uva.es
}

Advanced drug-delivery systems (DDSs) have been designed to transport pharmaceuticals to target tissues and to control drug release by responding to certain stimuli. Thus, DDSs will improve therapeutic effects, reduce drug toxicity and improve the release profile with respect to the administration of free drugs, and in some cases can even penetrate into the targeted cells if required. Stimuli-responsive systems have become increasingly important in drug delivery due to their ability to rapidly respond to specific stimuli or to changes in their environment, including $\mathrm{pH}$ [5], temperature, ion concentration [6], light [7] and other changes [8]. Different materials, including natural or synthetic polymers, can be used to design such stimuli-responsive systems. Natural polymers such as proteins also exhibit several characteristic advantages when used as a biomaterial to form drug carriers as they are able to form nanostructures according to their established secondary, tertiary and quaternary structures. Secondary structures comprise those such as an $\alpha$-helix or $\beta$ sheet that can be used to bind micro- and nanostructures together $[9,10]$. They have a versatile structure because of the broad range of design possibilities conferred by the high number of possible combinations of amino acids. In addition, proteins are made by living organisms and therefore have a higher likelihood of resembling natural tissues and being degraded by physiological pathways into the corresponding non-toxic byproducts. However, natural proteins vary from one batch to the next and can contain impurities, contaminants and infectious agents or cause hypersensitivity 
reactions. Genetic engineering has overcome these disadvantages and has allowed the creation and improvement of new bioinspired protein-based polymers with enhanced structural and functional features with respect to their natural analogous. As such, these polymers are excellent candidates for the design of drug-delivery systems. Examples of genetically engineered well-developed drug carriers include protein polymers composed from elastin-like polypeptides due to their thermoresponsive behaviour and ability to coacervate.

Elastin-like recombinamers (ELRs) are synthetic proteinaceous polymers bioinspired by natural elastin sequences and obtained using recombinant DNA techniques, thus allowing strict control of their amino acid composition and subsequent properties [11]. ELR genes are commonly expressed in Escherichia coli and purified by several heating and cooling cycles followed by centrifugation, in a process known as inverse temperature cycling (ITC).

ELRs are based on the recurrence of short repeating peptide motifs present in tropoelastin; one of the most common repetitions is the pentapeptide sequence (Val-Pro-Gly-XaaGly), where Xaa is any amino acid except L-Proline. This type of polymer has a wide range of interesting properties, most of them derived from natural elastin, such as their biocompatibility, excellent mechanical properties, ability to self-assemble [12] and stimuli-responsive behaviour [13]. Moreover, these polymers are able to respond to different environmental stimuli, such as $\mathrm{pH}$, ion concentration, pressure or temperature [8], with the latter being the most widely studied variable. The transition temperature $(\mathrm{Tt})$ is the characteristic temperature below which the polymer chains remains hydrated, disordered and relatively extended, and above which the ELR chain folds hydrophobically and assembles to form a separate phase. In this state the chains adopt a dynamic secondary structure known as a $\beta$-spiral [12, 14]. This $\mathrm{Tt}$ is controlled by intrinsic parameters, including composition, molecular weight and hydrophobicity, and it is also influenced by extrinsic factors such as concentration, solutes or $\mathrm{pH}$. The tailor-made design of ELRs enables their transition temperature to be tuned and adapted for specific applications.

ELRs have been used in a wide variety of applications in various biomedical fields such as drug delivery [15], tissue engineering [16], regenerative medicine [17, 18] and other medical devices [19]. The purpose of this review is to collect information about ELR-based delivery systems that promote target delivery, subsequently focusing on those drug carriers in which release is triggered by $\mathrm{pH}$ or temperature stimuli, especially depots and hydrogels that act as drug reservoirs and others not based on ELRs that have recently found uses as smart drug-delivery systems.

Three different types of ELR constructs can be distinguished based on the different strategies used to design targeted drug-delivery systems. Firstly, ELR-drug recombinant fusion constructs, in which therapeutic sequences are fused to the backbone of the ELR in what is known as ELPylation. This system allows both expression and purification to be enhanced while improving drug delivery and pharmacokinetics [20]. The second type of targeted drugdelivering ELRs consist of chemically bioconjugated recombinamers. In this case, modification is achieved by introduction of a "cross-linking" site in amino acid residues of the ELR in order to fuse a drug, thus allowing it to be delivered at the appropriate site and improving its pharmacokinetic properties (3). The final strategy described is based on drug encapsulation by ELR-based nanoparticles. A wide range of encapsulating ELRs have been studied, among which block copolymers that can form micelles by self-assembly in response to different parameters $(27,33 \mathrm{~A})$, chemically cross-linked microgel capsules and other systems used in gene delivery (37) can be highlighted.

With respect to drug delivery triggered by temperature, the thermosensitive characteristics of ELRs make them one of the most interesting systems as regards a response to pathological changes in temperature. Hyperthermia, or local heating of the tumour site, is an excellent stimulus for the development of new anti-cancer therapies as a result of the vasodilatation [21], vascular permeability [22] and increased intracellular traffic into the tumour cells [23]. These effects may allow successful drug delivery by ELR-based systems as they are thermoresponsive and capable of changing from a soluble state to an insoluble structure with the formation of aggregates or self-assembly into well-defined nanoparticles at the disease site. Thus, these smart architecture features have been applied in the biomedical field specifically for drug-delivery purposes. Very little research has been devoted to the development of smart $\mathrm{pH}$-sensitive ELRs, with recent proposals being related to ELR-based micelles with an ability to self-assemble and with pH-responsiveness [24] and pH-mediated drug delivery based on ELRs with an acidlabile linker [25].

One of the suggestions for controlling the release of therapeutic agents and avoiding a fluctuating saw-tooth-like release is to apply a depot or hydrogel system that acts as a support and reservoir in which the drug can be stored [26]. The role of this system is to allow controlled delivery of the drug that can be tuned as a result of the different features derived from material design as well as polymer and drug concentrations, inter-solubility or matrix degradation simultaneous with drug release.

Depot formation is governed by the smart behaviour and temperature sensitivity of ELRs; this, along with a tailormade structure designed to possess a $\mathrm{Tt}$ below the physiological temperature, makes them injectable systems. Thus, the ELR solution is injected at the site of action as a soluble ELR (unimers), and the polymers spontaneously coacervate to form the "drug depot" as a consequence of the temperature. In particular, regenerative medicine, neuroinflammation and cancer and diabetes therapy have benefited from this thermoresponsive behaviour [27]. Depending on the designed structure of the ELRs, a more organized polymeric network with a 3D structure can be obtained in hydrogels in which interchain connections can be provided by physical or chemical linkages. The interconnected 3D structure of the hydrogel, together with its ability to contain a large amount of water inside, provides a friendly environment for encapsulating therapeutic agents. Indeed, injectable hydrogels formed by in situ polymer crosslinking are of increasing interest for drug delivery as they enable a depot to be directly formed in vivo in the target tissue with just an injection and avoiding surgical 
implantation [28-30]. Thus, direct application of the ELR at the target site helps to extend the local drug exposure time while minimizing systemic side effects [31]. Depending on the complexity of the ELR structure and the nature of the crosslinking, several different physical and chemical hydrogels will be described highlighting their different applications resulting from the specific bioactive molecule released.

Finally, an overview of drug-delivery systems other than those based on ELRs will be provided. There are a wide variety of structures for drug-delivery systems made up of both polymeric and non-polymeric building blocks [32]. Some examples of natural proteins used in delivery systems, such as silk and collagen, will be described together with non-polymeric carriers defined by non-repetitive amino acid motifs with an ability to acquire tertiary or quaternary structures, including vault proteins [33] and viral proteins [34]. Subsequently, and staying with synthetic polymers, applications of poly(lactic-co-glycolic acid) (PLGA) in drug delivery will be reviewed [35], finishing with non-polymeric systems such as metal organic frameworks (MOFs) and their applications.

\section{TARGETED DRUG DELIVERY MEDIATED BY ELASTIN-LIKE RECOMBINAMERS}

Elastin-like recombinamers (ELRs) have been widely engineered to be suitable for application in targeted drug delivery without the need for any type of sensitivity to ensure drug release. Three different classes can be established based on the different strategies designed for this purpose, namely ELR-drug recombinant fusion constructs, ELR-drug combinations chemically bioconjugated in their monomeric and soluble form, and drug encapsulation by nanoparticle-forming ELRs.

\subsection{ELR-drug fusion constructs}

Recombinant DNA techniques have enormous potential for the construction of therapeutic proteins fused to an ELR backbone, which is often called ELPylation (for elastin-like polypeptide) [20]. This approach allows us to take advantage of a purification method that relies on the transition temperature described above, in which the recombinamer can be obtained pure after several heating and cooling cycles following by centrifugation, in a process called inverse temperature cycling (ITC). This system permits expression and purification to be enhanced while improving drug delivery and pharmacokinetics [36].

With this premise, an ELR was engineered fused to a polyaspartic acid tail (named ELR-D) with the aim of achieving self-assembled nanoparticles whose size is regulated by the charge repulsion of the aspartic acid tails. In order to add a biofunctionality to the nanoparticles formed, EGF (epidermal growth factor) was fused to the construct (to form the so-called ELR-D-EGF), being displayed on the surface of ELR-D. The binding of EGF to its receptor on the cell surface was assessed upon culture with A549 cells, a human lung adenocarcinoma epithelial cell line that overexpresses the EGF receptor, thereby confirming the expected increase in cell proliferation upon EGF binding when compared to the non-EGF-fused control. This system was also shown to be a good candidate for drug delivery applications by the loading of paclitaxel, a well-known anti-cancer drug, which showed a better internalization into A549 cellsinducing cell death [37].

In another study, a novel strategy involving fusion of a single-chain vascular endothelial growth factor $\left(\mathrm{VEGF}_{121}\right)$ derived polypeptide to an elastin-like recombinamer was designed with the aim of enhancing the purification and in vivo stability of growth factors. This method was denominated as purification and delivery system (PADS) and the fusion product as veGFPADS. The ELR-VEGF protein was successfully purified by ITC and evaluated in vitro in human umbilical vein cell (HUVEC) culture by means of vascular tube formation and cell proliferation and migration, showing results significantly comparable to free VEGF, thereby confirming the bioactive properties of the chimera and suggesting that the ELR tag has no effect on the binding of VEGF 121 to its receptor. This approach also led to slower plasma clearance of the growth factor when injected in mice (half the rate for free $\mathrm{VEGF}_{121}$ ), although biodistribution was different for each one, with the former mainly being located in the liver and the free growth factor in the kidney. This work shows the potential of fusing growth factors to ELRs in order to lower the purification cost while maintaining their bioactivity [38].

Likewise, taking advantage of the ability of some ELRs to aggregate into coacervates when the temperature is raised above the transition temperature, a keratinocyte growth factor (KGF)-ELR chimera was recombinantly designed and bioproduced. Subsequent use of this material in vivo for the treatment of chronic wounds in diabetic mice led to enhancement of the granulation and re-epithelization of the wound when compared with the effect of KGF entrapped in fibrin gels due to the sustained administration of KGF in the ELR coacervate [39].

In order to improve the treatment of dry eyes, lacritin (Lacrt), a protein component of human tears with prosecretory activity in the lacrimal gland, was fused to an ELR comprising the VPGVG pentapeptide, thus allowing localization of the drug at the site of action due to precipitation of the recombinamer at physiological temperature, above the $\mathrm{Tt}$, and overcoming the limitation of rapid tear turnover of single Lacrt when tested in vivo [40]. Another approach involving this same system combined the ability of a block copolymer (see following section concerning encapsulation systems) consisting of hydrophilic (serine-containing) and hydrophobic (isoleucine-containing) elastin-derived domains, thus being able to form nanoparticles at $37{ }^{\circ} \mathrm{C}$, with the therapeutic properties of lacritin, which led to a faster regeneration of the corneal epithelium after wound induction in non-obese diabetic (NOD) mice when compared to an ELR-Lacrt that does not assemble into particles [41]. Similarly, the knob domain of the fibre capsid protein of adenovirus serotype 5, previously bound to an ELR and confirmed to improve internalization into hepatocytes [42], which was shown to bind to the coxsackievirus and adenovirus receptor highly expressed in the lacrimal gland, was fused to the latter ELR-Lacrt nanoparticle, thus allowing selective transcytosis of the ELR-drug fusion into the cells of this gland [43]. 
The recombinant fusion of anti-inflammatory proteins, such as antibodies that recognize pro-inflammatory cytokines, has also been achieved using the above-mentioned ELPylation approach. Due to the limitations of prokaryotic recombinant expression systems regarding post-translational modifications that most of these anti-inflammatory proteins undergo, many of them have been expressed in plants. Some of the first studies performed in this bioproduction system were aimed at evaluating the expression and purification of different proteins fused to ELRs in tobacco leaves, with satisfactory results $[44,45]$. With regard to antibodies, antihuman TNF was also fused to an ELR, produced in Nicotiana tabacum and purified by ITC. Further investigation was performed both in vitro and in vivo and showed both similar bioactivity to free TNF and an increase from 28 minutes to 11 hours (24-fold) in terms of serum half-life and clearance, thus indicating an improvement in the pharmacokinetics of the antibody [46]. Similarly, IL-10 [47] and an HIV-neutralizing antibody [48] were also engineered into an ELR-bound DNA vector for expression in tobacco plants, showing analogous bioactivity to that of the free proteins without the need for cleavage from the ELR tag. Nevertheless, the use of peptides instead of whole proteins for fusion to ELRs allows the expression in more common systems such as E. coli, thereby reducing costs and usually performing better in terms of yields. As an example, six repetitions of the peptide AP1, a ligand for IL-4 receptors (IL-4Rs), were genetically bound to a VPGVG-based ELR. The resulting chimeric recombinamer was fluorescently labelled and found to bind to IL-4Rs in vitro in different tumour cell lines in which IL-4Rs are highly expressed, thus leading to an amplification of the expression of some antiapoptotic proteins and preventing drug-induced cell death. The system tested also resulted in tumour accumulation in vivo, with this effect being evident as early as 10 minutes post-injection of the ELR-AP1, peaking at $6 \mathrm{~h}$ and being maintained up to $24 \mathrm{~h}$ [49]. This carrier should therefore be tested as an actual drug-delivery system with a chemotherapeutic agent.

Alternatively, self-assembled ELRs have been designed as nanovaccines against tuberculosis by the recombinant fusion of an antigen from Mycobacterium tuberculosis. In this case, a nanoparticle-forming di-block consisting of a glutamic acid (E) hydrophilic block and an isoleucine (I) hydrophobic one, with different amino acids as guest residues, was chosen. The resulting particles were found to be monodisperse and stable despite fusion to the antigen, and additional work was performed in order to assess the ability of the nanovaccine to elicit an immune response in vivo, thus resulting in the triggering of an innate response following by the presence of IL-5 and IgM and IgG, which clearly suggests an adaptive Th2 response. Meanwhile, the ELR itself did not have any immunomodulatory effect. In conclusion, these results suggest the potential application of ELRs as antigen carriers [50].

\subsection{Chemically bioconjugated ELR-drug constructs}

Covalent bioconjugation has been extensively used to achieve therapeutic elastin-like recombinamers chemically fused to drugs, thus allowing an improvement in the solubility of hydrophobic drugs and in the pharmacokinetics of these drugs. This covalent binding is performed by introducing cross-linking sites via the modification of reactive groups in the side chains of different amino acids, such as thiol groups in cysteine or the $\varepsilon$-amino group in lysine residues. In contrast to the recombinant fusion, this approach implies that non-protein drugs can be bioconjugated.

Doxorubicin (Dox), a widely used chemotherapeutic agent, has been bioconjugated to several ELRs. One of the first studies in this regard accomplished such conjugation by modifying the amine groups present in lysine residues of the ELR to obtain a free maleimide group, which reacted with a Dox-hydrazone complex containing an acid-labile hydrazine bond to achieve bioconjugation. Further in vitro studies performed to assess the cytotoxicity and subcellular localization of the ELR-Dox showed a similar level of squamous cell carcinoma ( $\mathrm{FaDu}$ ) cell death (less than $10 \%$ of survival after $72 \mathrm{~h}$ ) when compared to the free drug, although different localizations were observed, with ELRDox being dispersed throughout the cytoplasm and free doxorubicin mostly internalized in the nucleus, suggesting different cell-death pathways in each case [51]. Another possibility involving chemical conjugation via cysteine residues was evaluated and an ELR-Dox drug system synthesized, this system allowing intracellular release of the drug as a result of an acid-labile hydrazine bond [52]. An alternative approach using the latter recombinamer involved the inclusion of a GFLG tetrapeptide linker that is recognized by lysosomal cathepsin proteases, thus permitting the release of the drug after endocytosis. A Tat domain, which is a cell-penetrating peptide (CPP) derived from the trans-activating protein found in HIV-1, was also fused to this recombinamer, theoretically improving cellular uptake of the conjugated drug. In vitro studies were performed with both non- and Dox-resistant carcinoma cell lines, resulting in a higher cytotoxicity towards drug-resistant cells in the case of the ELR-Dox when compared to the drug itself (1.4\% and $31.9 \%$ resistance, respectively, in the case of breast cancer cells) [53]. Additionally, a nanoparticle-forming block copolymer ELR was used to conjugate doxorubicin via cysteine residues. Hence, 40nm particles were achieved above the Tt of the ELR, with little effect of Dox on this feature and the drug being located at the core of the nanoparticle. Tumour regression was observed in in vivo assays using a mouse colon cancer model after a single dose, which represents a great advantage with respect to the free drug [54]. Furthermore, other chemotherapeutic drugs, such as paclitaxel, have been bioconjugated and found to present the same benefits as Dox in terms of pharmacokinetics and improved solubility [55].

As an example of a likely ELR as a drug carrier for maternal delivery and prevention of foetal exposure, an ELRfluorescent probe system was designed in order to demonstrate the potential of this class of polypeptides for use in such applications, starting from the hypothesis of the lower ability of these proteins to cross the placental barrier compared to free drugs. The covalent binding of a fluorescent dye to an ELR or a SynB1-ELR backbone, with SynB1 being another CPP, allowed traceability when injected intravenously into a pregnant rat model. The ex vivo fluorescent measurement of the placenta and the foetus showed that very small quantities of the labelled ELR crossed the placenta, whereas the observation of different 
maternal organs showed a preferential accumulation of the ELR in kidneys, liver and the placenta itself, thereby suggesting the potential of elastin-like recombinamers as safe drug carriers for different diseases, such as preeclampsia [56].

\subsection{Elastin-like recombinamers as non-responsive encapsulation systems}

The aforementioned recombinant nature of ELRs permits a strict control over their physical and chemical properties, thus allowing the tailoring of their self-assembly into different structures, such as different types of nanoparticles, depending on the amino acid present as guest residue in the VPGXG pentapeptide. One of the most widely explored possibilities is the construction of ELRs consisting of hydrophobic and hydrophilic blocks, known as "block copolymers". The design of a di-block recombinamer implies that it undergoes a conformational change from an extended form to micelles above the $\mathrm{Tt}$ of the hydrophobic part $[57,58]$. In this case, the $\mathrm{Tt}$ is called the critical micelle temperature (CMT) and can be controlled by varying the length and composition of the hydrophobic block, while the size of the micelle depends mostly on the length of the ELR and the hydrophilic-to-hydrophobic block ratio. The core of the micelle is suggested to be occupied by the hydrophobic block, while the hydrophilic one is oriented towards the corona, in contact with the surrounding water molecules. Further investigation regarding the self-assembly behaviour of ELRs into micelles or hollow vesicles (see ¡Error! No se encuentra el origen de la referencia.) involved changing the arrangement and length of the constituent blocks, leading to the conclusion that these two different structures can be obtained using ELRs comprising glutamic acid- and alaninebased blocks, thereby suggesting the potential functionalization of these recombinamers for subsequent applications [59]. Additionally, the effect of surfactants [60] and different salts [61] on ELR self-assembly was assessed and it was found that these conditions can completely modify the structure of the self-assembled ELRs, i.e. the formation of micelles or vesicles, depending on the concentration of these additives in the aqueous recombinamer solution.

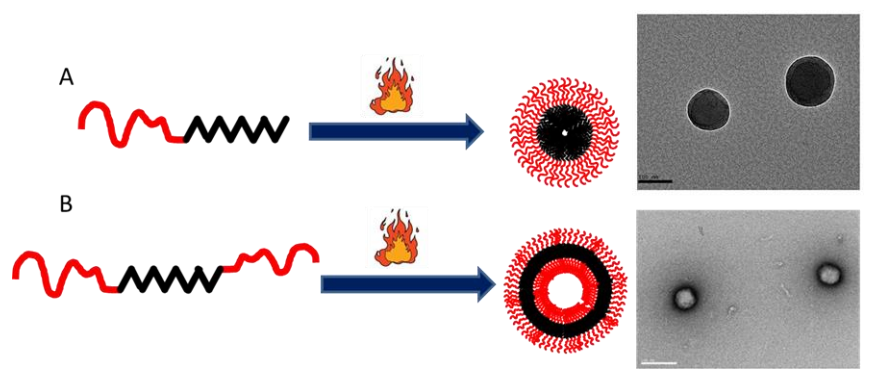

Figure 1. TEM images of self-assembled nanoparticles stained with $1 \%$ uranyl acetate of the ELR enriched with glutamic and alanine. Micelle like (A) and vesicle like selfassembled ELR architecture (B) [59].

With the purpose of exploring the possibility of adding novel bioactivities to ELRs in order to enhance their drug-delivery features, Plasmodium falciparum FK506-binding protein (FKBP), which binds to the chemotherapeutic antiproliferative drug rapamycin, was fused to the hydrophilic part of a di-block micelle-forming recombinamer. This modification increased the affinity of the ELR for this drug since FKBP is exposed in the corona of the nanoparticle. In

vitro studies in a breast cancer cell line showed an increase in the loading capacity of the drug by the particles and its release, therefore improving its activity towards these tumour cells [62].

Chemical cross-linking can be used as another method for the development of thermo-responsive microgel capsules (MCs) with potential applications in drug delivery. This approach permits shape-stable microspheres, the pore size of which can be reversibly tuned by increasing or lowering the temperature, thereby modifying the permeability to drugs, to be obtained. In a first example of this technology, a mixture of ELR and albumin at different ratios was cross-linked, with the resulting system showing shape stability and changes in the surface structure of the MCs depending on whether the temperature was above or below the Tt. In the first case, the release of BSA and prednisone acetate, both used as model drugs, was faster than in the latter. Optimal release was found in the temperature range $20-40{ }^{\circ} \mathrm{C}$ [63]. ELR/BSA MCs were subsequently produced by a two-step crosslinking method. The ability of these microcapsules to release a drug was evaluated using two similar fluorophores, namely rhodamine B (red) and FITC (green), this latter possessing a negative charge, thus showing a charge-independent release of both molecules from the MCs. Similarly to the previous work, release of the cargo molecules was faster when the temperature was raised above $\mathrm{Tt}$, and vice versa [64].

Dual microparticles consisting of two different biomaterials, namely ELR-chitosan or alginate-chitosan, have also been designed as a proof-of-concept methodology following a layer-by-layer approach and found to have potential as drugdelivery systems [65]. Further studies with the ELR-chitosan microparticles involved recombinant functionalization of the ELR with the RGD cell-adhesion sequence for integrins. These particles, with a size of 3-4 $\mu \mathrm{m}$, were loaded with fluorescently labelled DQ-ovoalbumin as a model protein and used to supplement the medium in human mesenchymal stem cells (hMSCs) culture for $72 \mathrm{~h}$. The findings of this study showed them to be non-cytotoxic, with improved internalization of the protein to the cytoplasm than in the case of the non-functionalized (non-RGD) ELR-chitosan microparticles, as evidenced by DQ-ovoalbumin degradation, which results in a shift in fluorescence emission. Thus, this strategy suggests the utilization of microparticles formed by different biocompatible macromolecules as carriers for drug delivery [66].

In a more recent work, elastin-like-silica hybrid micelles were obtained by fusing the silaffin R5 peptide to an ELR. Silaffins are polycationic peptides with affinity for silica found in Cylindrotheca fusiformis, playing a role in the formation of its silica cell walls. However, on their own these peptides self-assemble into uncontrolled structures, thus leading to a silicification process resulting in a network of fused silica nanoparticles. The fusion of this peptide to an ELR capable of self-assembling into micelles permits the synthesis of uniform hybrid ELR and silica nanoparticles that could be used as drug-delivery systems, as shown by the higher monodispersity and larger silica-coated micelles of 
the silaffin-containing recombinamer when compared with the micelle-forming non-silaffin counterpart [67].

The same group that engineered the ELR-D-EGF fusion protein discussed above designed another approach for loading of the previously described single-chain vascular endothelial growth factor ( $\left.\mathrm{scVEGF}_{121}\right)$-derived polypeptide into nanoparticles. This approach takes advantage of the noncovalent interaction between coiled-coil leucine zipper domains, one with acid (helix A) and another one with basic (helix B) residues, both peptides being designed to form stable, parallel, coiled-coil heterodimers (Figure 2). An ELR-D-helixB and a helixA-scVEGF ${ }_{121}$ were designed and recombinantly produced in $E$. coli and found to form coiledcoil nanoparticles. Further experiments involving the loading of paclitaxel into the nanoparticles showed the enhanced cytotoxicity of the resulting drug-delivery system upon supplementing the medium with these particles in the culture of HeLa cells. This study showed uptake of these particles by the cells and the release of paclitaxel specifically via the VEGF receptor [68].
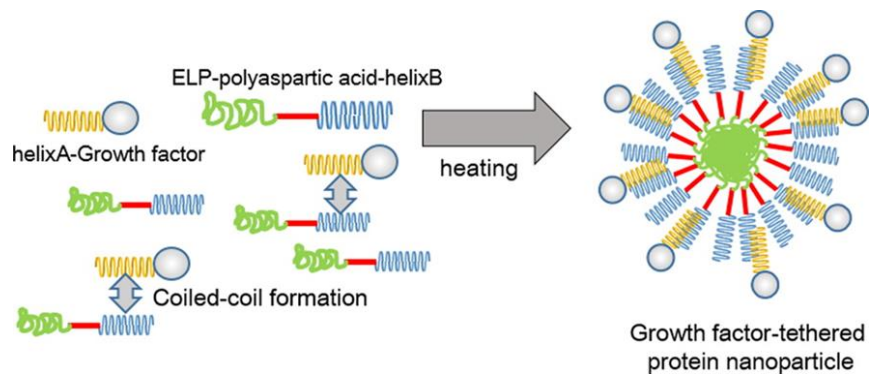

Figure 2. Schematic representation of the tethering of the growth factor to nanoparticles through the formation of coiled-coil assemblies. Reprinted with permission from Assal et al. [68].

Other novel encapsulation systems have been designed recently taking advantage of the properties of other recombinamers resulting from combination of the VPGAVG hexapeptide, also found in human elastin, and VPGVG sequences, together with crosslinking regions that permit further modification of the ELR. This ELR was found to form microparticles $(11 \pm 5 \mu \mathrm{m})$ via a water-in-oil emulsion, which were combined with and covalently attached to superparamagnetic iron oxide nanoparticles with the aim of applying a controlled magnetic field once implanted in vivo and improving the targeting and tracking of these vectors when loaded with a drug. To assess the actual potential of these microparticles, they were loaded with either fluorescently labelled bovine serum albumin (BSA) as a model drug or neural growth factor (NGF) for in vitro release studies. In the latter study, a neural differentiation of the PC12 cell line was also observed. However, this study was quite limited and some improvements are still required, especially a reduction in particle size in order to be suitable for in vivo applications [69].

Various studies have also been performed with ELRs as gene-delivery systems, with all of them relying on the electrostatic interactions between negatively charged DNA molecules and positively charged ELRs due to substitution of the guest amino acid in the VPGXG pentapeptide by an amine-containing residue, such as lysine, or the fusion of a tail including positively charged amino acids. The latter approach has been used to attach an oligolysine tail (K8) to the ELR to give the so-called K8-ELR, which was subsequently incubated with plasmid DNA (pDNA) including the gene encoding for green fluorescent protein (GFP) and found to exhibit good complexation and stability of the polyplexes. The transfection efficiency was also assessed by way of culture with in vitro MCF-7 breast cancer cells, with fluorescence due to GFP expression being observed. However, some cytotoxic effects were also observed, probably due to the K8 tail [70]. In another recent work, a positively charged ELR was fused to the penetratin cell-penetrating peptide and the LAEL fusogenic peptide. Stable polyplexes of around $200 \mathrm{~nm}$, with a zeta-potential of $+24 \mathrm{mV}$ were formed and found to be optimal for the desired application. Haemocompatibility and cytotoxicity assays showed the innocuous nature of these ELRs. Furthermore, the LAEL-containing ELR was found to be the best choice in terms of cellular uptake and transfection among the ELRs tested. These results show the potential of highly biocompatible ELRs for gene-therapy applications [71].

\section{DRUG DELIVERY TRIGGERED BY ELR SMART BEHAVIOUR}

As stated above, soluble ELRs suffer a reversible phase transition in response to changes in temperature which, depending on their architecture, causes them to aggregate or self-assemble into well-defined nanoparticles. As a result, these smart architectural features have been applied in the biomedical field, especially for drug-delivery purposes.

\subsection{Drug delivery triggered by temperature: hyperthermia and cancer therapy}

\subsubsection{ELR aggregates}

The use of drug delivery systems for cancer treatment is dependent on the enhanced permeability and retention (EPR) effect present in tumour tissues. The EPR effect is characterized by the accumulation of macromolecules and lipids as a consequence of restricted lymphatic drainage. The regional blood flow to the tumour, the permeability of the tumour vasculature, the structural barriers imposed by perivascular tumour cells and extracellular matrix, together with intratumoural pressure, must all be taken into account in the design of novel drug-delivery agents [72]. The combination of an EPR with local mild hyperthermia (40-44 $\left.{ }^{\circ} \mathrm{C}\right)$ [73] makes these constructs appropriate candidate biomaterials for cancer treatment.

The design of ELRs with a $\mathrm{Tt}$ similar to that found under conditions of mild hyperthermia opens the way to this application as, when local hyperthermia is applied, the ELR changes its soluble state to an insoluble one. The ELR therefore aggregates under local mild hyperthermia at the site of action and can be used for cancer therapy. This aggregation increases its accumulation and time of action. Tumour accumulation may be due to the passive trafficking of the ELR due to the EPR effect [27], the increased tumour blood flow and vascular permeability as a consequence of hyperthermia [74], or due to the aggregation itself, which localizes more ELR in the tumour region [75, 76]. Studies with a ${ }^{14} \mathrm{C}$-labeled ELR $\left(\left[{ }^{14} \mathrm{C}\right]\right.$ ELP1) allowed the degradation rate and accumulation in the tumour to be 
studied [75]. The in vitro degradation rate upon exposure to murine serum was $2.49 \mathrm{wt} . \% /$ day, with similar levels being obtained after intravenous injection into nude mice (2.46 wt.\%/day), with a terminal half-life of nearly 9 hours. These data showed a 1.8 -fold increase in accumulation for heated tumours when compared with controls. Another study investigated the enhanced accumulation of ELRs at the tumour site due to their thermosensitive behaviour [76]. Consecutive heating cycles were applied to the tumour, resulting in the formation of adherent ELR microparticles in the tumour vasculature in each thermal cycle. In contrast, when the heat was turned off the particles were dissolved, thereby creating a transvascular concentration gradient which pushed the ELR into the tumour space. Aggregate accumulation was monitored by labelling the ELR with fluorescent reporter molecules. This study resulted in a new methodology known as "tumour thermal cycling", which could potentially be used for targeted delivery to solid tumours.

At a cellular level, the ELR transition can be used to increase cellular uptake of the ELR-drug complex, thus triggering higher delivery levels of the drug. In order to achieve better results, short functional motifs known as cell-penetrating peptides (CPPs), such as penetratin, Bac, TAT and SynB1, were used. These CPPs have been shown to efficiently internalize various molecular loads in order to target the inside of eukaryotic cells [77-79]. Thus, Raucher's lab developed a thermally responsive ELR with the CPPs penetratin (pen), Tat or Bac fused to the inhibitor c-Myc (H1), which is known to inhibit proliferation in MCF-7 breast cancer cells [80]. The intracellular distribution of the resulting ELR-H1 fused with the different CPPs was evaluated. Bac-ELP-H1 was found to be localized in the nucleus and was the most potent inhibitor of MCF-7 cell proliferation at day 7. In contrast, the Pen- and Tat-modified ELRs were localized in the cytoplasm. As a result, Pen-ELP$\mathrm{H} 1$, which decreases the proliferation rate in MCF-7, had no effect on proliferation up to 11 days of treatment. This study combined the advantages of thermal targeting and delivery of a therapeutic peptide such as c-Myc, thus making this system an improvement on previous studies. Further in vivo research using an orthotopic model of breast cancer in C57BL/6 mice to test the ability of the Bac-ELR-H1 construct to target and reduce tumour growth was developed [81]. Treatment involving daily injections of Bac-ELR-H1 during 7 days over a total of 2 weeks, in combination with hyperthermia, reduced the progression of breast tumours by nearly $70 \%$. In treated mice the ELR was able to target the site of the solid tumour, escape from the vasculature, enter the tumour cells and inhibit their proliferation. Similar results were obtained with the same Bac-ELR-H1 construct but this time with glioblastoma in a rat glioma model [82]. An $80 \%$ reduction in tumour volume was observed, with complete regression in $80 \%$ of animals, when combined with hyperthermia. As a result, tumour-related neurological deficits were delayed and the median survival time doubled.

Additionally, a Bac peptide fused to an ELR was also used by the same group in further studies. Thus, addition of a Bac peptide at the $\mathrm{N}$-terminus and an amino acid fragment from a peptide derived from $\mathrm{p} 21$ at the $\mathrm{C}$-terminus led to the construct Bac-ELR-p21 [83]. Under mild hyperthermia conditions, the selected ELR showed a decrease in the proliferation of SKOV-3 cells in comparison with the thermally unresponsive control. This increase in the antiproliferation effect was due to the action of $\mathrm{p} 21$, which was located in the nucleus for those cells incubated under hyperthermia. Another polypeptide was designed using the same ELR structure but with the CPP SynB1 at the Nterminus and the therapeutic peptide GRG at the C-terminus (SynB1-ELR-GRG) [84]. GRG was designed to interfere with UsnRNP biogenesis by competing for methylation with $\mathrm{Sm}$ proteins. In vitro assays in HeLa cells showed the localization of the polymer in the cytoplasm. Cellular uptake, proliferation and apoptosis effects derived from the therapeutic GRG peptide were therefore increased by hyperthermia treatment.

Similarly, a targeted CPP-ELR has been used in combination with hyperthermia to deliver small molecule drugs such as doxorubicin [85] or paclitaxel [86]. Thus, doxorubicin was conjugated to the Tat-ELR via a cathepsin-cleavable GFLG spacer to form the construct Tat-ELR-GFLG-Dox as explained above. In a subsequent study [85], the cytotoxic effects of the drug increased 20-fold when the ELR aggregated as a result of the application of mild hyperthermia. Paclixatel, in turn, was linked to a SynB1ELR via an acid-cleavable hydrazone linker [86], being SynB1 a CPP that is known to internalize via endocytosis. The hydrazone linker is cleaved at the acidic $\mathrm{pH}$ found in lysosomes and endosomes, thereby triggering the intracellular release of paclixatel. In this study the delivery of paclixatel was achieved under hyperthermia, and inhibition of proliferation and apoptosis induction was confirmed using MCF-7 breast cancer cells and found to be comparable with the effects of the free drug.

\subsubsection{Self-assembled ELR particles}

In addition to thermosensitive ELR constructs that aggregate, even reaching depot status, self-assembled ELR-based architectures are also attractive for the biomedical field. In this sense ELRs are usually designed as block copolymers comprising a hydrophilic and a hydrophobic part being able to self-assemble, thus leading to the formation of welldefined nano-sized particles. When the polymer is solubilized in aqueous solution and is heated above its $\mathrm{Tt}$, the ELR chains orientate in a way that triggers the formation of core-corona structures, with the corona comprising hydrophilic blocks and core hydrophobic blocks. The micelle or hollow vesicle shape resulting after self-assembly can be changed by varying the ELR block arrangement and length [59]. Studies with different ELR constructs containing glutamic acid and alanine blocks confirmed this possibility, thereby highlighting the high versatility of ELR-based diblocks. ELR diblocks in which the hydrophobic part possesses a $\mathrm{Tt}>42{ }^{\circ} \mathrm{C}$ under local mild-hyperthermia conditions have been widely studied due to their potential use in cancer therapy [87].

Self-assembled ELR nanoparticles can acquire different functionalities upon the incorporation of recognition motifs such as RGD, NGR or Fn3 peptides in the hydrophilic block. The RGD peptide acts by increasing the avidity of the copolymer for its ligand ( $\alpha$ IIB integrin in activated platelets and the $\alpha v \beta 3$ integrin in healthy angiogenic tissue in addition to tumour vasculature) while maintaining its $\mathrm{Tt}$ and ability to 
self-assemble, thereby constituting a thermally tunable switch for the ELR in K562 leukaemia cells [88]. NGR is known to bind to CD13 isoforms expressed in tumour vessels and has been widely used for tumour targeting. This peptide was bound to ELR diblocks in a series of constructs with different hydrophilic-to-hydrophobic ratios [89]. At ratios of between 1:2 and 2:1, monodisperse spherical micelles were found with the peptide pointing outwards. In addition, the critical micelle temperature (CMT) could be controlled by varying the length of the lower Tt block. This study was the first demonstration of temperature-triggered multivalency in which ELR diblocks with a functional peptide maintain their ability to self-assemble and target tumour cells, with self-assembly into micelles enhancing the local density of the functional peptide on the corona.

The fibronectin type III domain (Fn3), which binds the integrin $\alpha v \beta 3$, has also been fused to ELR diblocks [90]. The self-assembly behaviour of the resulting constructs was studied through the bioproduction of ELRs with different hydrophilic and hydrophobic block lengths. The result was an increase in the uptake of ELR with Fn3 in comparison with the unimers in a transfected human leukaemia cell line overexpressing the integrin $\alpha \mathrm{v} \beta 3$ (K562/ $\alpha \mathrm{v} \beta 3)$. In a later study, a qualitative breakthrough in the design of ELR diblocks fused to functional peptides was accomplished. Thus, Chilkoti's group constructed an ELR-based drug carrier termed nanopeptifier, that was able to improve cellular uptake by controlled exposure of CPPs, which toggled in response to the application of external mild hyperthermia (Figure 3) [91]. The construct (Arg8-ELRBCcBH3) was genetically engineered to possess a CPP (Arg8) at the $\mathrm{N}$-terminus and the therapeutic payload peptide $\mathrm{cBH} 3$ (derived from the proapoptotic Bak protein) located at the Cterminus. The therapeutic $\mathrm{cBH} 3$ peptide was separated by an RVRR peptide linker cleavable by furin and cathepsin B proteases. The nanopeptifiers are soluble at physiological temperature $\left(37{ }^{\circ} \mathrm{C}\right)$, whereas under of mild hyperthermia conditions $\left(42{ }^{\circ} \mathrm{C}\right)$ they self-assemble into spherical micelles displaying a high density of CPP on their corona and with $\mathrm{cBH} 3$ located in the micelle core. Although some uptake of the nanopeptifier occurs at $37{ }^{\circ} \mathrm{C}$, when the temperature is increased to $42{ }^{\circ} \mathrm{C}$ this cell uptake increases dramatically. Once the complex is internalized, the action of either furin (localized in the trans-Golgi) or cathepsin B (early and late endosomes and lysosomes) favours release of the $\mathrm{cBH} 3$ load. Increases of up to 130 arbitrary cell fluorescence units were found to induce the therapeutic effect of $\mathrm{cBH} 3$. Its therapeutic action was assessed by activation of caspase-3, which is involved in apoptotic processes. Thus, the nanopeptifier was able to create a cytotoxic switch that induces apoptosis only when it is in the micelle conformation.

In addition to ELRs genetically designed as block copolymers, a series of smart ELR-PEG block constructs have been synthesized chemically [92]. The specific strain promoted alkyne-azide cycloaddition (SPAAC) reaction was used to build the copolymer. Constructs with different ELR and PEG lengths were accomplished by varying their architecture (linear or miktoarm). The result was conservation of the thermosensitive nature of the ELR, which promoted self-assembly into micelles. The ability of these constructs to encapsulate a hydrophobic fluorescence
A
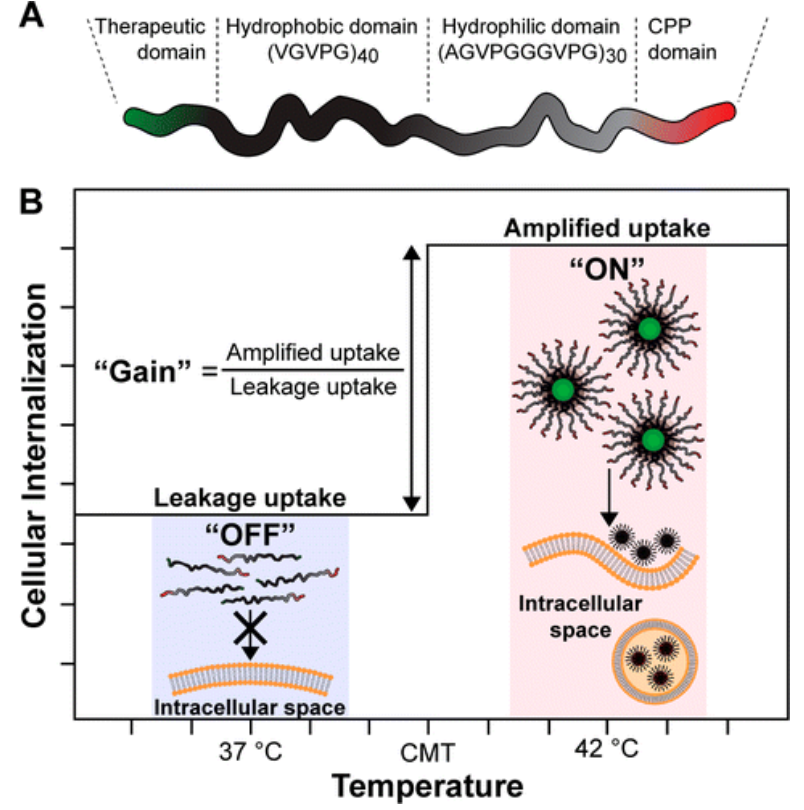

Figure 3. Design and function of an ELR diblock nanopeptifier. (A) Each nanopeptifier is formed by a therapeutic payload, an ELR diblock and a CPP. (B) At physiological temperature $\left(37^{\circ} \mathrm{C}\right)$ "off state", nanopeptifiers are soluble displaying a single CPP on their hydrophilic terminus. At mild hyperthermia conditions $\left(42^{\circ} \mathrm{C}\right)$ "on state" the nanopeptifiers self-assemble into spherical micelles, displaying a high density of CPPs on the micelle corona. The increases CPP density amplifies the cellular uptake [91].

dye was shown, thereby demonstrating the potential of these ELR-PEG hybrid copolymers for the encapsulation of hydrophobic drugs.

The above examples reflect the potential of ELRs as thermoresponsive materials, which are of growing interest in the biomedical field. Their recombinant nature, which allows a total control of their architecture, and their self-assembly properties open the way to new features such as thermal switching, which can even be used to direct cellular uptake. All these studies involve new applications in diseases such as cancer and are therefore a promising field for ELR-based biomaterials.

\subsection{Drug-delivery systems based on pH-sensitive ELRs}

pH-Sensitive drug-delivery systems have received increasing interest in recent years as $\mathrm{pH}$ plays an important role in many biological process and some diseases such as asthma, peptic ulcer, heart disorders, hypertension and cancer [93]. For example, the $\mathrm{pH}$ found in healthy tissue or in the blood is different to that found in tumours due to tumour hypoxia and the production of substances such as lactic acid resulting from anaerobic metabolism and ion channel changes [94, 95].

Given these differences in $\mathrm{pH}$ between normal tissue and the tumour microenvironment, Callahan et al. proposed the first pH-sensitive ELR-based polypeptide micelle that disassembles at the lower $\mathrm{pH}$ found in solid tumours. Based on previous work in which these authors demonstrated that ELR block copolymers with different lower critical solution temperatures (LCSTs) are able to form micelles over a 
specific temperature range [89], ELR $\mathrm{BCS}_{\mathrm{BC}}$ containing histidine-rich hydrophobic blocks were designed with the aim of creating $\mathrm{pH}$-sensitive nanoparticles that self-assemble at $\mathrm{pH} 7.4$ and disassemble at $\mathrm{pH} 6.4$ [24, 96]. Thus, they synthesized two different ELR $_{\mathrm{BC}}$, containing a combination of hydrophobic ( $\mathrm{pH}$-insensitive block, to form the corona of the micelles) and hydrophilic blocks ( $\mathrm{pH}$-sensitive blocks, to form the core of the micelles). [VG7A8]-80/[VH4]-100 was selected as it was found to be the only ELR $_{\mathrm{BC}}$ that formed stable nanoparticles at $37{ }^{\circ} \mathrm{C}$. A pH-insensitive ELR [VG7A8]-64/[V]-120 was selected as negative control in order to verify the ability of the $\mathrm{pH}$-sensitive micelle formed by [VG7A8]-80/[VH4]-100 to respond to changes in $\mathrm{pH}$.

The ELR $\mathrm{BC}_{\mathrm{BC}}$ were examined in nude mice bearing HCT-15 human colon adenocarcinoma xerographs. Both polymers maintain the beneficial behaviour of high MW drug-delivery nanoparticles, with $\left[\mathrm{VG}_{7} \mathrm{~A}_{8}\right]-80 /\left[\mathrm{VH}_{4}\right]-100$ presenting a lower accumulation in healthy tissues and greater affinity for the lower $\mathrm{pH}$ of the tumour environment. It was found that [VG7A8]-80/[VH4]-100 shows a more homogeneous spatial distribution and greater penetration within the tumour tissue compared to $\mathrm{pH}$-insensitive $\mathrm{ELR}_{\mathrm{BC}}$ micelles based on

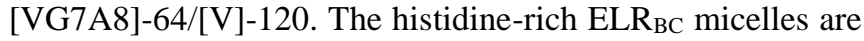
able to undergo a $\mathrm{pH}$-triggered disassembly that means the individual polymer chains increase their diffusion capacity and can penetrate inside the tumour. However, $\mathrm{pH}$ insensitive micelles tend to accumulate around the tumour space as they cannot respond to lower $\mathrm{pH}$, and cannot therefore undergo $\mathrm{pH}$-dependent disassembly, thus meaning that the rate of accumulation in the extravascular space increases. This new $\mathrm{pH}$-responsive mechanism suggests the potential utility of this system for improving drug delivery to, or the imaging of, solid tumours.

Another strategy is based on the design of a different structure in which $\mathrm{pH}$-dependent delivery is related to $\mathrm{pH}$ labile amino acid sequences. In order to improve the results obtained by MacKay et al. in a previously reported study [54], a new strategy was proposed by $\mathrm{Hu}$ et al. Thus, these authors reported the first ELR genetically fused to a tumour homing peptide, F3, fused to the same structure proposed previously by MacKay et $\mathrm{al}$. and also carrying a cysteinerich fragment (Gly-Gly-Cys) 8 (C8) with the aim of creating a bioactive and well-defined polypeptide F3-ELP-C8, which was conjugated with doxorubicin (DOX). F3 is a 31 -amino acid sequence that specifically attaches to nucleolin, which is present on the surface of activated lymphocytes, angiogenic endothelial cells and many tumour cells [97, 98]. The C8 segment was selected to bind multiple DOX molecules to the Cys residues of the F3-ELR-C8 via an acid-labile hydrazine linker (3-maleimide propionic acid hydrazine (BMPH)) to form F3-ELR-C8-DOX (Figure 4). They demonstrated that DOX has a faster delivery at lower $\mathrm{pH}$ (5.0) than at $\mathrm{pH} 7.4$ (physiological $\mathrm{pH}$ ), where most of the DOX molecules are attached to the conjugate. This result reveals that the hydrazone bond is stable at the $\mathrm{pH}$ of blood and healthy tissues but breaks at lower $\mathrm{pH}$. The $\mathrm{pH}$-dependent release of DOX from the conjugates suggests endolysosomal drug delivery once the nanoparticles have been taken up by the cells, which is related to the $\mathrm{pH}$-responsive behaviour.

In vitro studies demonstrated that F3-ELR-C8-DOX nanoparticles bound to the cell surface earlier and exhibited more drug accumulation in the cell cytoplasm and nucleus than ELR-C8-DOX, thereby demonstrating the important role that F3 plays in the cell interaction. This result was confirmed by way of in vivo experiments in mice with a C26 tumour model, in which F3-ELR-C8-DOX was found to have a longer half-life than free DOX and ELR-C8-DOX, a lower accumulation in cardiomyocytes and did not cause histological damage in heart tissue. This is an important point due to the fact that cardiocytotoxicity is often the worst side effect of DOX use. With regard to its efficiency in reducing the tumour volume, the results obtained thirteen days after treatment demonstrated that it exhibits better antitumour efficacy and increased animal survival compared with the control ELR-C8-DOX. Furthermore, histological analyses showed a higher apoptotic effect, which is related to the pH-sensitivity and local tumour distribution of the nanoparticles around the tumour cells [25].

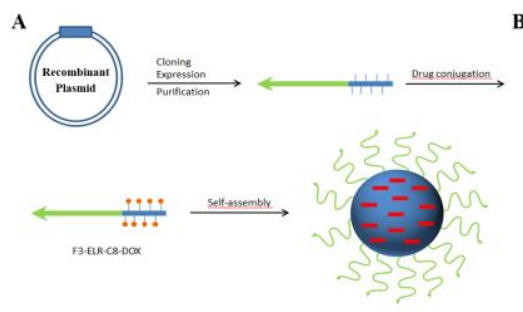

B

Figure 4. Schematic representation of the synthesis of F3ELR-C8-DOX nanoparticles, showing (A) the synthesisconjugation of F3-ELR-C8-DOX and (B) the molecular structure of this drug delivery system. Adapted with permission from Hu et al. [25].

\section{THERAPEUTIC ELR DEPOTS AND HYDROGELS}

The smart behaviour and temperature sensitivity of ELRs can be exploited in such a way as to control their aggregation both in vitro and in vivo, thus paving the way to advanced medical applications such as drug depot formation for tissue regeneration, neuroinflammation, cancer and diabetes.

As a general rule of thumb, these ELRs have been designed to possess a Tt below the physiological temperature of $37^{\circ} \mathrm{C}$. The ELRs are solubilized and injected at the desired site of action as soluble ELRs (unimers), where they immediately coacervate and form the depot as a result of the temperature. Thus, the direct application of ELRs at the target site helps to extend local drug exposure time while minimizing systemic side effects [31].

As stated above, one of the main applications for ELR depots is in regenerative medicine. Thus, research in knee joint regeneration has investigated the use of a thermally responsive ELR that could spontaneously aggregate upon injection to form a "drug depot". The in vivo biodistribution in a rat model showed that the aggregated ELR had an approximately 25 -fold increase in intra-articular half-life in comparison with the non-aggregated ELR. This finding made it appropriate for sustained delivery of protein drugs for treating osteoarthritis [99]. More recent work in the treatment of post-traumatic arthritis involved the development of a cross-linked ELR and the evaluation of intra-articular delivery of IL1Ra (IL1 receptor antagonist) and TNFRII (TNF $\alpha$ inhibitor). The results showed a sustained delivery of both inhibitors and a corresponding 
decrease in osteoarthritic damage caused by the disease [100]. As regards the development of carriers for the delivery of osteogenic drugs, such as bone morphogenetic protein (BMP)-2 and BMP-14, a VPAVG-based ELR that forms stable and monodisperse depots was used to encapsulate both therapeutic proteins separately. The in vitro release profile was found to be fast during the first 24 hours followed by a slower release over 14 days. Transdifferentiation of $\mathrm{C} 2 \mathrm{C} 12$ mouse myoblast cells towards osteoblasts in in vitro cell culture experiments demonstrated the efficient delivery of both BMPs. These results suggest the application of this system in the delivery of many other drugs [101].

ELR depots can also be applied in other pathological targets such as neuroinflammation in order to improve the inflammatory symptoms. In studies to treat local neuroinflamation at the dorsal root ganglion using ELR depots, a sevenfold increase was found in perineural half-life in comparison with the soluble ELP. This result provides evidence of high and sustained levels of therapeutics at the dorsal root ganglion while minimizing systemic exposure [102]. Another interesting approach in the growing field of neuroinflamation is the use of depots comprising ELRs chemically conjugated to curcumin (a TNF $\alpha$ antagonist) via a degradable carbamate linkage [103]. The resulting ELR maintained the thermally-triggered depot formation after curcumin conjugation and hence the bioactivity against $\mathrm{TNF} \alpha$. When injected proximal to the sciatic nerve in mice via intramuscular (i.m.) injection, ELR-curcumin underwent a thermally triggered soluble-insoluble phase transition, thus leading to in situ formation of a depot and resulting in a fivefold higher level of the drug at four days post-injection when compared with the free drug and an up to sevenfold decrease in plasma levels.

ELR-radionuclide conjugates have been explored as therapy for the local treatment of tumours. In this case, an ELR was designed and conjugated to the therapeutic agent iodine-131 and delivered intratumourally. An increase in the residence time in the tumour, together with a longer protection of the radionuclide from halogenation, when compared with a soluble ELR, was observed [104]. Although these ELRiodine-131 depots improved survival and tumour regression in murine xenograft tumours, they were unable to achieve complete tumour regression. Further optimization was performed to improve this aspect [105]. This optimization was achieved after investigating the effect of a wide range of parameters that influence tumour retention and modulate the physical properties of the depot, such as molecular weight, amino-acid composition and the tyrosine-rich peptide appended to the carboxy-terminus for radiolabelling. The radionuclide-polypeptide resulting from this study was able to self-assemble to form seeds that led to prolonged intratumoural retention, tumour growth delay in $100 \%$ of tumours and cure in more than $67 \%$ of tumour-bearing animals in two human xenografts of hypopharyngeal and prostate cancer cells (FaDu and PC-3 respectively).

ELR depots can also be used to deliver the glucagon-like peptide-1 (GLP-1), with which they form a $50 \mathrm{kDa}$ polymer that has shown great promise for the treatment of type 2 diabetes with a glucose-dependent insulinotropic effect. Following this strategy, ELR depots were genetically designed with the incorporation of GLP-1 flanked by protease cleavage sites [106]. The delivery of GLP1 from the depot and activation of the GLP-1 receptor were subsequently confirmed. The resulting depot was termed a "protease operated depot" (POD). The novel contribution of this system lies in the reduction in the number of injections since a single injection of GLP1-ELR depot reduced blood glucose levels in mice for up to 5 days, which is 120 times longer than for an injection of the native peptide.

Different ELRs hydrogels have been designed for biomedical applications with an amphiphilic block corecombinamer structure that spontaneously self-assemble to form gels which physical crosslinking relying on hydrophobic interactions between apolar blocks. These systems mimick in vivo cellular environment enabling in vitro studies of cell-ECM interactions [107]. Being made up of ELRs constitutes and advantage because of their tunable mechanical properties, well defined biological and chemical activity and, if necessary, a controlled topography [108, 109]. These features made them potentially useful as scaffolds for tissue engineering. The only drawback with such non-covalent bonding is that it sometimes results in inadequate mechanical properties or low stability of these structures. To overcome these disadvantages and to stabilize ELR-based hydrogels, a bioinspired peptide such as the silk fibroin domain [110], which is able to establish more robust physical cross-links, has been incorporated into the ELR backbone to give rise to the family of silk-elastin-like polymers (SELPs). This family of genetically engineered protein block copolymers is made up of silk fibroin domains, which follow patterns such as GAGAGS, and elastin-like blocks of the type GXGVP, where the guest amino acid " $\mathrm{X}$ " can be any amino acid except proline. The silk-like block tends to self-assemble into an anti-parallel $\beta$-sheets secondary structure, thereby providing physical crosslinking sites for the SELP polymeric systems that enable access to physical hydrogels with higher thermal and chemical stability and mechanical tunability [111]. Exhaustive studies have been conducted into the modulation of SELP properties, especially by varying the silk-elastin ratio [112], molecular weight [113] and X residue in the elastin sequence [114]. SELPs can act as an injectable gel-forming material for controlled drug and gene release of DNA under physiological conditions. Initial research by Gustafson et al. [112] studied the release from hydrogels comprising various SELPs with different silk-elastin ratios and loaded with adenoviruses carrying reporter genes for head and neck tumours. The biopolymer SELP-815K, with eight silk and fifteen elastin units and a lysine (K)-modified elastin, had the highest ratio of silk content and showed the highest gene expression compared with the others studied (SELP-415K and SELP-47K). The tumour to liver expression ratio was up to 55-fold higher for the SELP-815K system compared to viral injection without the polymer, thus proving the utility of these matrices for gene therapy in head and neck cancer. Subsequent studies [115] with SELR matrices consisting of the same structured SELP (SELP-815K) loaded with an adenovirus containing both thymidine kinase- 1 and luciferase genes was performed to check the efficacy and safety of tumour therapy. The SELP-815K delivery system was injected intratumourally into a mouse model of head and neck cancer and the gene transfection efficiency, duration of 
transgene expression and biodistribution analyzed using the bioluminescence provided by the luciferase gene. SELPcontrolled therapeutic agent delivery at the tumour site resulted in greater confinement, with no detectable spread to the liver, which contrasts with the results obtained for intratumoural injection of adenoviruses in saline, in which 50\% of animals showed clear liver dissemination. An approximately fivefold greater reduction in tumour size was observed in the adenovirus-SELP $815 \mathrm{~K}$ system compared with the results obtained for saline/adenovirus treatment, thus demonstrating the anticancer efficacy of these hydrogel systems [115].

SELP structures modified to introduce sensitivity to matrixmetalloproteinases (MMPs) were designed to increase the local delivery of bioactive agents to solid tumours in which MMPs are overexpressed. A hydrogel that is degradable by MMPs was produced and its protease sensitivity tested in vitro by incubation with different metalloproteinases (MMP2 and MMP-9) to assess complete removal from the potential administration site and the correct release of bioactive agents. This was checked by measuring the mechanical properties of the hydrogel and the fluorescence of polystyrene beads released, with the results suggesting the ability of these systems to respond to local changes in MMP concentration when injected intratumourally [116]. In vivo degradation assays were subsequently performed in the presence of solid head and neck tumours. A significantly higher degradation was observed for MMP-responsive SELPs than for SELP-815K, whereas degradation of their hydrogel devices is polymer structure dependent, thus demonstrating the tunable nature of MMP-responsive SELPs for matrix-mediated gene delivery [117]. In light of the above, we can conclude that this type of biomaterial is especially useful for gene therapy as polymeric hydrogels control DNA release, allow localized delivery to specific or damaged tissue and protect DNA from degradation, thereby improving the safety and efficacy of gene delivery.

ELRs have been used to form ELR-collagen hydrogels in order to optimize and reinforce the features of pure collagen hydrogels. One of the most widely applied hydrogels for local drug delivery applications is that comprising collagen, despite the fact that it lacks the rigidity required for tissue engineering and, more importantly, that it is rapidly degraded by collagenases to form products that elicit inflammation. Anderson and co-workers have prepared ELPcollagen hydrogels physically cross-linked by hydrogen bonding and hydrophobic associations that displayed superior physical and mechanical properties than pure collagen scaffolds, which was attributed to a homogeneous network structure with additional reinforcement due to the ELR aggregates [118]. Subsequent studies concerned the release behaviour of the ELR-collagen hydrogels prepared in the presence of doxycycline hyclate and loaded with different amounts $(1-5 \%, \mathrm{w} / \mathrm{w})$ of this commonly used broad-spectrum antibiotic in periodontal or acne therapy. The ELR-collagen composite showed slower doxycycline release compared to the collagen hydrogels, probably due to a smaller swelling ratio, with a gradual time-dependent drug release over a period of 5 days irrespective of the hydrogel composition. The collagen and ELR that make up the hydrogels did not interfere with the bioactivity of the drug and the released doxycycline was effective against four bacterial strains (E. coli, P. aeruginosa, S. sanguinis and methicillin-resistant Staphylococcus aureus), thus meaning that this biocompatible composite hydrogel allows sustained release of an antibiotic at the local site with the potential to minimize post-surgical infections and support wound healing.

A novel ultrasound-based method has been used to generate physical ELR hydrogel scaffolds for the local delivery of proteins and antibiotics. These hydrogels are formed by sonicating an aqueous solution of the recombinamer [(VPGVG $\left.)_{120}\right]$ for a short period of time at low temperature $\left(4^{\circ} \mathrm{C}\right)$ and subsequently incubating at physiological temperature for an extended period of time, in the presence of bovine serum albumin and doxycycline hyclate, which were added to the sonicated ELR to be enclosed. The mechanism suggested for physical cross-linking involves a hydrophobic interaction between polymeric chains after ultrasonication. The release of BSA and doxycycline from the scaffold was studied at different temperatures, above (37 $\left.{ }^{\circ} \mathrm{C}\right)$ and below $\left(25^{\circ} \mathrm{C}\right)$ the transition temperature $(\mathrm{Tt})$ and with different loading concentrations of both bioactive molecules. Both BSA and doxycycline showed a gradual time-dependent release that is affected by factors such as drug molecular weight and loading concentration. The highlight of this approach is the ease of formation of ELR hydrogels without needing to add chemical crosslinking agents and a release governed by different factors such as temperature or drug loading dose, thereby suggesting potential applications in controlled drug delivery [119].

To increase the structural stability and mechanical properties of ELR hydrogels, which may be necessary for some applications, chemical hydrogels were synthesised by chemical, enzymatic or photo-irradiated cross-linking [120123]. In addition, a chemical hydrogel to be used as an injectable "click" system has been developed starting from a pair of elastin-like recombinamers suitably chemically modified in such a way that a covalently crosslinked hydrogel is formed under physiological conditions, in a short period of time, and once the two components are in contact. The inclusion of an amino acid that can subsequently be modified to contain adequate functional groups for covalent cross-linking is necessary for this purpose. The introduction of a lysine residue as a guest amino acid distributed along the peptide chain allows ELRs to be modified at the $\varepsilon$-amino group to bear the reactive groups necessary for the "click reaction" [11]. To this end, ELRs were modified to carry azide and cyclooctyne groups capable of generating interchain bonds through triazol heterocycles via a copperfree 1,3-dipolar cycloaddition. The resulting hydrogel system is biocompatible and permits the homogeneous entrapment of drugs and bioactive molecules [124]. Nanogels were prepared at different temperatures by mixing dilute solutions of the aforementioned click-reactive ELRs with the aim of understanding the relation between the macroscopic properties of the resulting ELR-click gels and the initial stages of the chemical crosslinking process, which involve fractal modes of gel formation. Different topographies were obtained for the nanogels prepared at temperatures below and above the molecular transition temperature of the ELRs, thus implying that the cross-linking temperature determines the microstructure of the final hydrogels [124]. In a subsequent study, the previously developed hydrogels 
obtained by the catalyst-free click reaction of elastin-like recombinamers were used to fabricate a new class of vascular stents coated with this biocompatible material. Thus, the bare metal stent is embedded into an injection mold that is filled with the ELR gel. In order to avoid atherosclerotic plaque formation that induces re-stenosis, stent endothelization was performed in a bioreactor under dynamic pressure and flow conditions. Once endothelialized, the ELR-covered stents constituted an endovascular device with a physical barrier against atherosclerotic plaque, biocompatibility, no inflammatory response and physiological haemocompatiblity, thus paving the way to a future generation of eluting stents [125].

One of the most advanced hydrogel-based constructs for drug delivery is made up of enzymatically cross-linked ELRs that are able to release bioactive substances under specific stimulus from the environment. As has been described previously [126], there is a correlation between the elastolytic activity and pathological conditions such as atherosclerosis, cystic fibrosis or chronic wounds, amongst others. In this regard, Bandiera et al. have designed an ad hoc ELR-based hydrogel system that carries both chemical cross-linking and protease-sensitive domains (Figure 5). ELR crosslinking is performed via transglutaminases in the presence of a recombinant green fluorescent protein (eGFP) that is embedded during the gelation process. The hydrogels formed were exposed to the proteolytic activity derived from $P$. aeruginosa and human polymorphonuclear leukocytes (PMNs), which triggered the gradual release of eGFP from the matrix as it was degraded. In short, a drug delivery platform capable of interacting with biological systems that can sense a chemical abnormality in damaged tissues and respond by releasing the therapeutic agent to interfere with the pathological process, thus leading to the inhibition of potentially harmful processes, has been designed [127].

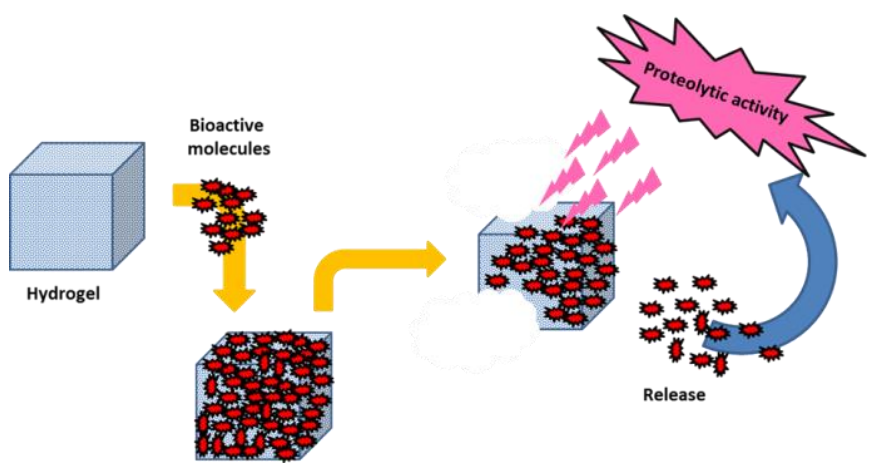

Figure 5. Bioactive molecule delivery triggered by elastolytic activity of pathological processes and drug release leading to proteolytic activity inhibition. Adapted with permission from Bandiera et al. [127].

An alternative approach to chemical hydrogels is that designed by Chilkoti et al., which consists of a reversible hydrogel that is formed under mild oxidative conditions by intermolecular disulfide chemical crosslinking of ELRs containing periodic cysteine residues along their chain. The rapid gelation of these custom-designed ELRs under physiological conditions upon addition of a low concentration of hydrogen peroxide $(0.3 \mathrm{wt} \%)$ makes them useful as an injectable gel-forming material. Preliminary in vitro studies of the release profiles for BSA protein indicated a sustained release pattern, thereby demonstrating the advantage of hydrogel formation for stable entrapment of a BSA payload with respect to the control ELR-based coacervates. Indeed, after 4 hours of incubation only $20-40 \%$ of release was detected for ELR hydrogels crosslinked via cysteine residues, whereas complete release was observed for ELR coacervates over the same time period. The in vivo intratumoural injection of in situ gelling radiolabelled ELR to mice bearing human pharynx squamous xenografts showed higher and more prolonged tumour retention (greater than $40 \%$ of the payload) and homogeneous distribution across the entire tumour, in contrast to ELR coacervates, for which retention was less than $10 \%$. This enhancement of radionuclide retention makes these systems suitable for local cancer therapy of tumours refractory to surgical removal.

\section{SMART DRUG-DELIVERY SYSTEMS NOT INVOLVING ELRS}

Numerous drug-delivery systems other than those based on elastin have been designed to achieve therapeutic efficiency at the application site and to overcome the aforementioned limitations in classical therapeutic disease treatments [2]. The most common such drug-delivery systems comprise new macromolecular carriers with a defined structure and properties that can respond to environmental stimuli with a pronounced change in their structure. To the best of our knowledge, these drug-delivery systems can be made from a wide variety of structures involving both polymeric and nonpolymeric building blocks [32]. One interesting improvement is the use of environmentally sensitive polymers that are able to respond to external stimuli such as temperature, $\mathrm{pH}$, ion concentration or electric fields, among others. A great variety of drug-delivery vectors have been designed using the above-mentioned building blocks, including micelles, liposomes, polymeric and protein drug conjugates or inorganic nanoparticles, each with different advantages $[3,4,128]$.

Amongst other polymeric drug-delivery systems consisting of natural proteins, those based on silk and collagen, which have been mentioned above in combination with ELPs, can be highlighted.

Silk proteins has been used with great success in devices for cell culture and tissue regeneration and have been approved by the FDA due to their versatility and biocompatibility $[129,130]$. Numata et al. have proposed a genetically engineered silk-based ionic pDNA complex with a poly(Llysine) that interacts with pDNA and has a higher content of tumour-homing peptide (THP) than in their previous studies in this field with the aim of improving its transfection efficiency and specificity for tumour cells [131]. Two different THPs were used with three different cell lines, namely MDA-MB-435 melanoma, MDA-MB-231 metastatic human breast tumour cells and MCF10A non-tumour mammary epithelial cells as control. The results showed that the pDNA complexes containing THP showed greater specificity against tumour cells compared with lipofectamine and the pDNA complex without THP used as control. In addition, a comparison of these THPs showed that the pDNA complex with F3 as THP appears to be the best candidate for target delivery due to its lower cytotoxicity as a result of the 
ability of pDNA to complex with Lyp1 to transfect nontumourigenic cells [132].

A very recent study describes the fabrication of silk fibroin (SF) microparticles for the delivery of small-molecule drugs for the treatment of arthritis by increasing the local residence time after intra-articular injection of the drug-loaded particles. To this end, microparticles were formed from a solution of a mixture of SF and the fluorophore $\mathrm{Cy} 7$, which establish mutual hydrophobic interactions, using previously described methods but with different ratios of the two components. Microparticle formation was confirmed by scanning electron microscopy, finding a wide range of particles that were nevertheless suitable for intra-articular injection. In vitro release studies revealed a faster release in the case of particles containing lower amounts of SF, thereby suggesting a negative effect of $\mathrm{Cy} 7$ on particle stabilization by diminishing intermolecular SF interactions. However, the release of Cy7 exceeded $10 \%$ of the loaded molecule over the $160 \mathrm{~h}$ that the experiment lasted. Additional experiments were performed in rats to evaluate intra-articular retention of the fluorophore using an in vivo imaging instrument. However, as Cy7 scattering and decay resulted in difficulties in measuring the evolution of the model compound over space and time, the decay half-life was used as the study parameter and showed an increase from $12.6 \mathrm{~h}( \pm 2.2 \mathrm{~h})$, in the case of the SF-Cy7 solution without particle-forming treatment to $43.3 \mathrm{~h}( \pm 14.4 \mathrm{~h})$ for the SF-Cy7 microparticles. Moreover, localization was improved with the novel treatment, thereby reducing the exposure of adjacent tissues to the fluorophore. All these findings suggest the need for further studies regarding this approach to intra-articular drug-delivery systems [133]. However, some problems arise from this work as the particles were not loaded with an actual drug or with non-hydrophobic molecules. Furthermore, fluorophore decay half-life might not be an accurate measurement of drug retention, although a slower decay was demonstrated when compared to SF-Cy7 solutions.

Collagen can be shaped into a great variety of forms for use as scaffolds or drug-delivery systems in numerous medical applications [134]. In order to prepare novel collagen (CG) scaffolds for use as drug-delivery systems that are likely to respond to an external stimulus, they were loaded with paramagnetic iron oxide nanoparticles (NPs) and subsequently covalently cross-linked by way of a dehydrothermal cross-linking treatment to obtain porous matrices. Different characterization techniques were used to gain information about the scaffolds themselves (e.g. SEM) and the amount of NPs included in them (thermogravimetric analysis). Fluorescein was subsequently used as a model compound to study the release by fluorescence spectrometry. The results showed a very low diffusion of fluorescein after immersion of the CGNP scaffold in water during 60 minutes when compared to the CG control. Furthermore, a controlled release of the compound was observed upon application of an external magnetic field to the NP-loaded system, and said release could be switched on and off by switching the magnetic field on and off. Finally, a cell viability assay was performed with the scaffold alone. This study showed a reduction of about $10 \%$ in cell survival in the case of both CG and CGNPs when compared to the negative control, which could be considered as a promising result for further application of this system [134].

As regards polymeric carriers for encapsulated drugs, numerous types of synthetic materials are currently employed, including poly(lactic acid) (PLA), poly( $\varepsilon$ caprolactone) (PCL), and poly(lactide-co-glycolide) (PLGA) [4]. Indeed, poly(lactic-co-glycolic acid) (PLGA) has been approved by the FDA for use in several therapeutic devices because of its ability to biodegrade, its biocompatibility and its multi-responsive behaviour [35, 135]. PLGA has found numerous applications, for example, in tissue engineering, medical and surgical devices and drug delivery [35]. Herein we focus our attention on applications of importance in drug delivery. Thus, numerous chemotherapeutic agents, such as docetaxel, paclitaxel, doxorubicin and other peptides, can be delivered using PLGA-based delivery systems [35]. Moreover, PLGA inmunoconjugates can be applied as targeted anti-cancer systems. Thus, Parveen $e t$ al. designed a PLGA-chitosan-PEG (polyethylene glycol) conjugated with paclitaxel, and in vitro studies carried out in retinoblastoma, invasive breast cancer and pancreatic cell lines demonstrated that PLGA-CS-PEG nanoparticles have a more potent antiproliferative effect and ability to induce cell cycle arrest than PLGA nanoparticles and free paclitaxel. These results suggest that the new conjugate with PEG and chitosan could be a future candidate for targeted delivery of chemotherapeutic agents [136]. Another targeted delivery system could be applied in the field of diagnostic imaging, as proposed by Niu et al. and Schleich et al. in recent studies $[137,138]$.

Collagen membranes are excellent candidates for guided bone regeneration (GBR) in combination with bone grafting materials (BGM) as they are subsequently absorbed, thereby avoiding the need for second surgery to remove the membrane [139]. However, as mentioned earlier, this system undergoes fast degradation, thereby limiting the possibility of the controlled release of drugs such as dexamethasone (DEX), which is an excellent osteoinductive growth factor [140-143]. Piao et al. have overcome this problem by incorporating PLGA, which is an excellent controlled release carrier that allows an efficient release of the drug [144, 145]. In vitro studies in MG63 cells revealed that release of DEX from PLGA on the collagen surface is faster by day 5 but can be controlled for four weeks. These results also show that PLGA improves the attachment of cells in the collagen membrane conjugate compared with the collagen membrane also. In vivo analyses have been carried out in rat models with a calvarial bone defect. The results obtained after eight weeks showed that the collagen membrane coated with has a better ability to induce osteogenesis than the membrane without DEX [146] due to controlled release from PLGA into the collagen membrane. DEX induces cell proliferation, differentiation, extracellular matrix secretion and tissue formation [147]. 
A new approach, namely the use of PLGA as a coating for drug-eluting stents, has recently been gaining importance, especially as atherosclerosis is one of the main problems related to stent implantation. In initial studies, Ganaha et al. had shown the ability of a combination of PLGA microspheres with a stainless steel stent and angiostatin to decrease plaque progression and avoid re-stenosis [148]. The favourable characteristics of PLGA, such as biocompatibility, biodegradability and its non-toxic properties, offer a wide range of possibilities for application in the development of new drug-delivery systems and other biomedical applications.

A novel proposal for the treatment of invasive breast cancer, consisting of a $\mathrm{pH}$-sensitive polymer conjugated with siRNA fragments, was developed by Kaushal et al. This system comprises a $\beta$-cyclodextrin core linked to the designer polymer by an acid-labile hydrazone bond, with the polymer itself comprising a hydrophobic monomer (HMA, hydrophobic hexyl methacrylate) connected to a $\mathrm{pH}$ sensitive monomer (DMAEMA, 2-(dimethylamino)ethyl methacrylate) and, in turn, to a cationic monomer (TMAEMA, N,N,N-trimethylaminoethyl methacrylate) (Figure 6). This latter cationic domain is attached to the antiRhoC siRNA by electrostatic interactions between the amine groups of the polymer and the phosphate groups of the siRNA. In vivo studies in SUM149 and MDA-MB-231 cell lines revealed that the resulting nanoparticles can be internalized into the cells, then leak from the endosome to subsequently deliver their siRNA load. Expression of the RhoC gene is selectively diminished by $90-100 \%$ and, as a result, the invasion, motility and migration of both SUM149 and MDA-MB-231 are considerably reduced. Other targets could be explored in the future with the aim of increasing the diversity of these smart nanoparticles combined with siRNA to reduce cell invasion and motility and induce an ability to reduce other types of tumour cells [149].

Other non-polymeric drug-delivery carriers are defined by non-repetitive amino acid motifs with an ability to form tertiary or quaternary structures, including vault proteins [33] and viral proteins, amongst other [34].

One example of vault proteins was proposed by Buehler et al. with the aim of improving their previous proposals for vault-drug delivery [33]. In this case, they formed a new class of nanoparticles resulting from the covalent attachment of NS5A1-31amphipathic $\alpha$-helix (a peptide derived from hepatitis C virus) to MVP, with these NPs showing a selfassembled protein shell with a lipophilic core that is able to retain small molecules for subsequent controlled drug delivery. Bryostatin 1 was subsequently incorporated into the AH-vault carrier with the aim of developing an antiHIV/AIDS agent. Previous studies showed that bryostatin 1 incorporated into lipid nanoparticles can activate latent HIV and can be triggered against CD4+ $\mathrm{T}$ cells [150]. The resulting $\mathrm{AH}$-vault-bryostatin 1 was found to activate latent HIV provirus in J-Lat 10.6 cells in a dose-dependent manner compared with empty AH-vaults, and was also able to prompt cell-surface expression of CD69 at similar concentrations to those required to activate latent HIV [151, 152]. In vivo analyses in $\mathrm{C} 57 / \mathrm{bl} 6$ mice revealed that over $90 \%$ of CD4+ had been induced to express CD69 24 h postinjection of $\mathrm{AH}$ vault with bryostatin 1, thus highlighting
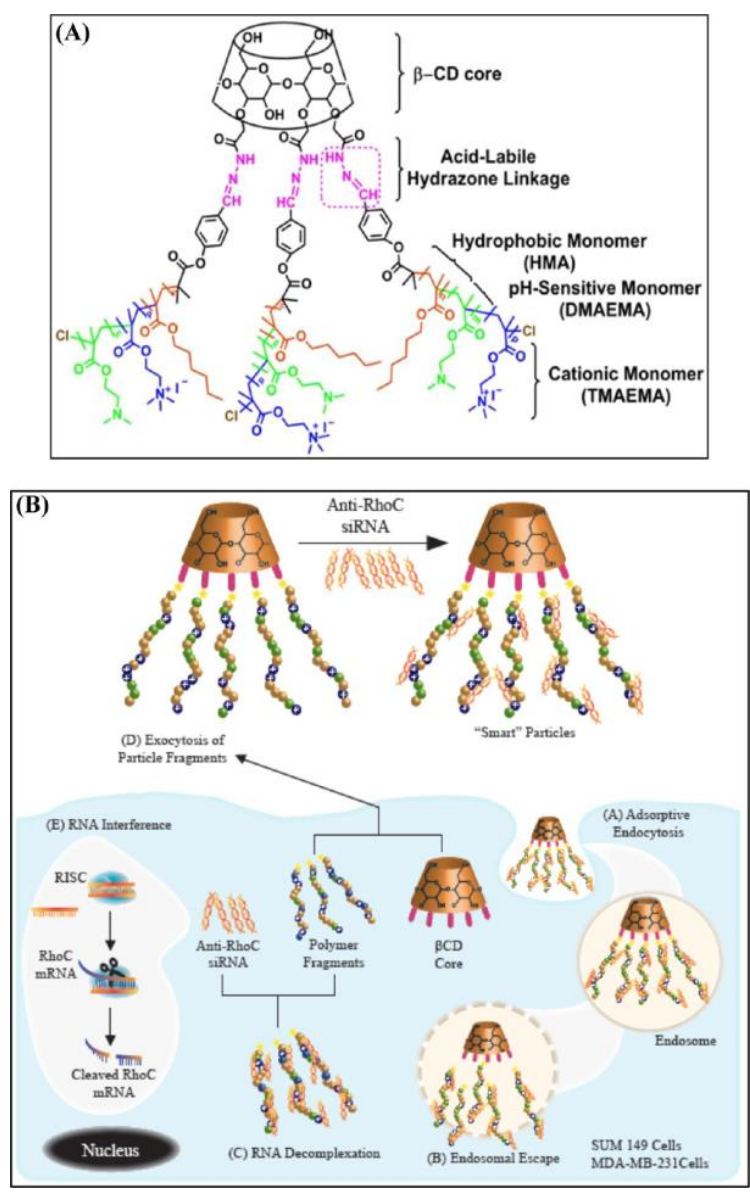

Figure 6. (A) Chemical structure of the $\mathrm{pH}$-sensitive, $\beta$-CDg-P(HMA79-co-DMAEMA33-co-TMAEMA48)4.8

polymer. (B) Schematic design showing condensation of anti-RhoC siRNA molecules by star-shaped $\mathrm{pH}$ sensitive polymers forming smart particles, which are internalized by endocytosis. In the endosome, acid-labile hydrazone linkages are hydrolyzed by the acidic $\mathrm{pH}$, which release the $\mathrm{P}$ (HMAco-DMAEMA-co-TMAEMA) grafts that rupture the endosomal membrane and release the loaded siRNA cargo into the cytoplasm. In the cytoplasm, anti-RhoC siRNA binds to RhoC mRNA triggering its degradation while particle debris is eliminated by exocytosis. Reproduced with permission from Kaushal et al. [149].

that excellent delivery of the drug can be obtained by combining both compounds.

Viral protein nanoparticles (VNPs) are another novel strategy for using non-polymeric nanocarriers as a drugdelivery system due to their excellent ability to mimic the natural process by which viruses penetrate cell membranes, with the added advantage that these nanoparticles are biocompatible and biodegradable. VNPs based on plant viruses and bacteriophages are excellent candidates as these viral proteins are not able to infect mammalian cells [153155].

The use of one of these plant viruses for drug delivery has been evaluated by Aljabali et al., who showed the ability of Cowpea mosaic virus (CPMV) to covalently conjugate with DOX in two ways, namely via a stabile amide bond (CPMVDOX) and a labile disulfide bridge (CPMV-SS-DOX). In 
vitro analyses in the HeLa cell line revealed that, at low dosage, CPMV-DOX is more effective for cell killing than free DOX because of its concentration-independent and time-delayed release. CPMV-DOX targets the endolysosomal compartment of HeLa cells and reduces the side effects of free DOX, such as the cardiotoxicity mentioned previously. The results for CPMV-SS-DOX revealed that this system exhibits a similar behaviour to DOX, possibly due to a more rapid dissociation of the disulfide-bridge, and therefore DOX delivery, prior to cellular uptake.

A further application of virus plant nanoparticles has been proposed by Esfandiari et al. Thus, a nanoparticle comprising potato virus $\mathrm{X}$ was conjugated with herceptin, a monoclonal anti-Her2/neu antibody that is used as a chemotherapeutic agent in breast cancer, via a chemical linker. In vitro flow cytometric studies in the SK-BR-3 and SK-OV-3 cell lines showed that, at different doses, PVXHer2 showed a greater apoptotic effect than the same doses of free herceptin [156].

Metal organic frameworks (MOFs) comprise a combination of metals connected to organic molecules via a linker. These systems are attracting increasing interest in biomedical applications due to their excellent ability to encapsulate and protect the drug release, thus making them good systems for drug delivery [157]. In order to demonstrate the controlled delivery of drugs by such systems, Adhikari et al. designed a $\mathrm{pH}$-responsive zeolitic imidazole framework (ZIF) containing zinc as the metal component and imizadole or another derivative as organic linker [158] with aim of encapsulating DOX within two different ZIFs. The resulting polymers showed an enormous difference in terms of their pH-responsiveness, with ZIF-8 demonstrating an efficient response capacity at acidic $\mathrm{pH}$, releasing $\mathrm{DOX}$ in the required manner. However, in the presence of liposomes and micelles, both ZIFs exhibited a high release rate, especially ZIF-8, probably due to the destabilization of their respective structures. The authors of this study demonstrated that drug release can be controlled without difficulty using different ZIFs and, optionally, liposomes [159].

\section{CONCLUSION}

ELRs have been extensively used for drug delivery in a variety of different forms, including soluble macromolecular carriers, self-assembled nanoparticles, cross-linked microparticles, thermally coacervated depots or crosslinked hydrogels. Targeted drug delivery using soluble macromolecular carriers encompasses different constructs, such as ELR-drug recombinant fusion constructs, ELR-drug systems chemically bioconjugated in their monomeric and soluble form, and drug encapsulation by nanoparticleforming ELRs. These constructs allow controlled release at the target site, thereby suggesting the potential of elastin-like recombinamers as safe drug carriers for use in the treatment of different diseases.

The reversible phase transition that ELRs undergo in response to changes in temperature allows the formation of structure-dependent architectures, such as aggregates or well-defined nanoparticles, and the use of such systems in the biomedical field, especially for drug-delivery purposes and even for direct cellular uptake. Particularly interesting examples of this can be found in cancer treatment, where the use of local mild hyperthermia and enhanced permeability retention has resulted in satisfactory treatments targeted directly at the tumour site.

The direct application of ELRs by injection as soluble monomers at the site of action to form depots extends drug exposure time while minimizing systemic side effects. This also paves the way to advanced medical applications, such as in tissue regeneration, neuroinflammation, cancer and diabetes. In addition, the use of ELR-based hydrogel systems that act as both a support and reservoir in which drugs can be stored results in a non-fluctuating and sustained drug release, thereby reducing side effects and increasing drug retention at the site of action with respect to the administration of free drugs.

\section{CONFLICT OF INTEREST}

The authors declare no competing financial interest.

\section{ACKNOWLEDGEMENTS}

We acknowledge financial support from the EU through the European Social Fund (ESF) and European Regional Development Fund (ERDF), from the MINECO (MAT201341723-R, MAT2013-42473-R, MAT2012-38043 and PRIPIBAR-2011-1403), the Junta de Castilla y León (Projects VA049A11, VA152A12, and VA155A12), the CIBER$\mathrm{BBN}$, the JCyL and the Instituto de Salud Carlos III under the Network Center of Regenerative Medicine and Cellular Therapy of Castilla and León.

\section{REFERENCES}

[1] Hench LL, Polak JM. Third-generation biomedical materials. Science 2002; 295(5557): 1014-7.

[2] Schattling P, Jochum FD, Theato P. Multi-stimuli responsive polymers-the all-in-one talents. Polymer Chemistry 2014; 5(1): 25-36.

[3] Zhu Y, Liao L. Applications of Nanoparticles for Anticancer Drug Delivery: A Review. Journal of Nanoscience and Nanotechnology 2015; 15(7): 4753-73.

[4] Yang Z, Gao D, Cao Z, et al. Drug and gene co-delivery systems for cancer treatment. Biomaterials Science 2015.

[5] Ribeiro A, Arias FJ, Reguera J, Alonso M, RodríguezCabello JC. Influence of the amino-acid sequence on the inverse temperature transition of elastin-like polymers. Biophysical journal 2009; 97(1): 312-20.

[6] Reguera J, Urry DW, Parker TM, McPherson DT, Rodríguez-Cabello JC. Effect of $\mathrm{NaCl}$ on the exothermic and endothermic components of the inverse temperature transition of a model elastin-like polymer. Biomacromolecules 2007; 8(2): 354-58.

[7] Alvarez - Rodríguez R, Arias FJ, Santos M, Testera AM, Rodríguez - Cabello JC. Gold Tailored Photosensitive Elastin - like Polymer: Synthesis of Temperature, $\mathrm{pH}$ and UV - vis Sensitive Probes. Macromolecular rapid communications 2010; 31(6): 568-73.

[8] Urry DW. Molecular machines: how motion and other functions of living organisms can result from reversible 
chemical changes. Angewandte Chemie International Edition in English 1993; 32(6): 819-41.

[9] Koehl P, Delarue M. Polar and nonpolar atomic environments in the protein core: implications for folding and binding. Proteins 1994; 20(3): 264-78.

[10] Elemans JAAW, Rowan AE, Nolte RJM. Mastering molecular matter. Supramolecular architectures by hierarchical self-assembly. Journal of Materials Chemistry 2003; 13(11): 2661-70.

[11] Testera AM, Girotti A, de Torre IG, et al. Biocompatible elastin-like click gels: design, synthesis and characterization. Journal of Materials Science: Materials in Medicine 2015; 26(2): 1-13.

[12] Urry DW. What sustains life?: consilient mechanisms for protein-based machines and materials: Springer Science \& Business Media; 2007.

[13] Li B, Alonso DO, Daggett V. The molecular basis for the inverse temperature transition of elastin. Journal of molecular biology 2001; 305(3): 581-92.

[14] Venkatachalam C, Urry D. Development of a linear helical conformation from its cyclic correlate. $\beta$-Spiral model of the elastin poly (pentapeptide)(VPGVG) n. Macromolecules 1981; 14(5): 1225-29.

[15] McDaniel JR, Callahan DJ, Chilkoti A. Drug delivery to solid tumors by elastin-like polypeptides. Advanced drug delivery reviews 2010; 62(15): 1456-67.

[16] Nettles DL, Chilkoti A, Setton LA. Applications of elastin-like polypeptides in tissue engineering. Advanced drug delivery reviews 2010; 62(15): 1479-85.

[17] Garcia-Arevalo C, Pierna M, Girotti A, Arias FJ, Rodriguez-Cabello JC. A comparative study of cell behavior on different energetic and bioactive polymeric surfaces made from elastin-like recombinamers. Soft Matter 2012; 8(11): 3239-49.

[18] Liu JC, Tirrell DA. Cell response to RGD density in cross-linked artificial extracellular matrix protein films. Biomacromolecules 2008; 9(11): 2984-8.

[19] Weber M, Gonzalez de Torre I, Moreira R, et al. Multiple-Step Injection Molding for Fibrin-Based TissueEngineered Heart Valves. Tissue Engineering Part C: Methods 2015.

[20] Floss DM, Schallau K, Rose-John S, Conrad U, Scheller J. Elastin-like polypeptides revolutionize recombinant protein expression and their biomedical application. Trends in biotechnology 2010; 28(1): 37-45.

[21] Dudar TE, Jain RK. Differential response of normal and tumor microcirculation to hyperthermia. Cancer Research 1984; 44(2): 605-12.

[22] Kong G, Braun RD, Dewhirst MW. Hyperthermia enables tumor-specific nanoparticle delivery: effect of particle size. Cancer research 2000; 60(16): 4440-45.

[23] Chen B, Zhou M, Xu LX. Study of vascular endothelial cell morphology during hyperthermia. Journal of Thermal Biology 2005; 30(2): 111-17.
[24] Callahan DJ, Liu W, Li X, et al. Triple stimulusresponsive polypeptide nanoparticles that enhance intratumoral spatial distribution. Nano letters 2012; 12(4): 2165-70.

[25] Hu J, Xie L, Zhao W, Sun M, Liu X, Gao W. Design of tumor-homing and $\mathrm{pH}$-responsive polypeptide-doxorubicin nanoparticles with enhanced anticancer efficacy and reduced side effects. Chemical Communications 2015; 51(57): 11405-08.

[26] Hoare TR, Kohane DS. Hydrogels in drug delivery: progress and challenges. Polymer 2008; 49(8): 1993-2007.

[27] Dreher MR, Liu W, Michelich CR, Dewhirst MW, Yuan F, Chilkoti A. Tumor vascular permeability, accumulation, and penetration of macromolecular drug carriers. J Natl Cancer Inst 2006; 98(5): 335-44.

[28] Hoffman AS. Hydrogels for biomedical applications. Adv Drug Deliv Rev 2002; 54(1): 3-12.

[29] Kopecek J. Hydrogel biomaterials: a smart future? Biomaterials 2007; 28(34): 5185-92.

[30] Van Tomme SR, Storm G, Hennink WE. In situ gelling hydrogels for pharmaceutical and biomedical applications. International Journal of Pharmaceutics 2008; 355(1-2): 1-18.

[31] Evans CH, Kraus VB, Setton LA. Progress in intraarticular therapy. Nat Rev Rheumatol 2014; 10(1): 11-22.

[32] Kopeček J. Smart and genetically engineered biomaterials and drug delivery systems. European Journal of Pharmaceutical Sciences 2003; 20(1): 1-16.

[33] Buehler DC, Toso DB, Kickhoefer VA, Zhou ZH, Rome LH. Vaults engineered for hydrophobic drug delivery. Small 2011; 7(10): 1432-39.

[34] Georgens C, Weyermann J, Zimmer A. Recombinant virus like particles as drug delivery system. Current pharmaceutical biotechnology 2005; 6(1): 49-55.

[35] Kapoor DN, Bhatia A, Kaur R, Sharma R, Kaur G, Dhawan S. PLGA: a unique polymer for drug delivery. Therapeutic delivery 2015; 6(1): 41-58.

[36] Rodríguez-Cabello JC, Piña MJ, Ibáñez-Fonseca A, Fernández-Colino A, Arias FJ. Nanotechnological Approaches to Therapeutic Delivery Using Elastin-Like Recombinamers. Bioconjugate Chemistry 2015; 26(7): 125265 .

[37] Matsumoto R, Hara R, Andou T, Mie M, Kobatake E. Targeting of EGF-displayed protein nanoparticles with anticancer drugs. Journal of biomedical materials research Part B, Applied biomaterials 2014; 102(8): 1792-8.

[38] George EM, Liu H, Robinson GG, Mahdi F, Perkins E, Bidwell GL, 3rd. Growth factor purification and delivery systems (PADS) for therapeutic angiogenesis. Vascular cell 2015; 7(1): 1 .

[39] Koria P, Yagi H, Kitagawa Y, et al. Self-assembling elastin-like peptides growth factor chimeric nanoparticles for the treatment of chronic wounds. Proceedings of the National Academy of Sciences of the United States of America 2011; 108(3): 1034-9. 
[40] Wang W, Jashnani A, Aluri SR, et al. A thermoresponsive protein treatment for dry eyes. Journal of controlled release : official journal of the Controlled Release Society 2015; 199: 156-67.

[41] Wang W, Despanie J, Shi P, et al. Lacritin-mediated regeneration of the corneal epithelia by protein polymer nanoparticles. Journal of materials chemistry B, Materials for biology and medicine 2014; 2(46): 8131-41.

[42] Sun G, Hsueh PY, Janib SM, Hamm-Alvarez S, MacKay JA. Design and cellular internalization of genetically engineered polypeptide nanoparticles displaying adenovirus knob domain. Journal of controlled release : official journal of the Controlled Release Society 2011; 155(2): 218-26.

[43] Hsueh PY, Edman MC, Sun G, et al. Tear-mediated delivery of nanoparticles through transcytosis of the lacrimal gland. Journal of controlled release : official journal of the Controlled Release Society 2015; 208: 2-13.

[44] Patel J, Zhu H, Menassa R, Gyenis L, Richman A, Brandle J. Elastin-like polypeptide fusions enhance the accumulation of recombinant proteins in tobacco leaves. Transgenic research 2007; 16(2): 239-49.

[45] Conley AJ, Joensuu JJ, Jevnikar AM, Menassa R, Brandle JE. Optimization of elastin-like polypeptide fusions for expression and purification of recombinant proteins in plants. Biotechnology and bioengineering 2009; 103(3): 56273.

[46] Conrad U, Plagmann I, Malchow S, et al. ELPylated anti-human TNF therapeutic single-domain antibodies for prevention of lethal septic shock. Plant biotechnology journal 2011; 9(1): 22-31.

[47] Kaldis A, Ahmad A, Reid A, et al. High-level production of human interleukin-10 fusions in tobacco cell suspension cultures. Plant biotechnology journal 2013; 11(5): 535-45.

[48] Floss DM, Sack M, Arcalis E, et al. Influence of elastin-like peptide fusions on the quantity and quality of a tobacco-derived human immunodeficiency virus-neutralizing antibody. Plant biotechnology journal 2009; 7(9): 899-913.

[49] Sarangthem V, Cho EA, Bae SM, et al. Construction and application of elastin like polypeptide containing IL-4 receptor targeting peptide. PloS one 2013; 8(12): e81891.

[50] Garcia-Arevalo C, Bermejo-Martin JF, Rico L, et al. Immunomodulatory nanoparticles from elastin-like recombinamers: single-molecules for tuberculosis vaccine development. Molecular pharmaceutics 2013; 10(2): 586-97.

[51] Dreher MR, Raucher D, Balu N, Michael Colvin O, Ludeman SM, Chilkoti A. Evaluation of an elastin-like polypeptide-doxorubicin conjugate for cancer therapy. Journal of controlled release : official journal of the Controlled Release Society 2003; 91(1-2): 31-43.

[52] Furgeson DY, Dreher MR, Chilkoti A. Structural optimization of a "smart" doxorubicin-polypeptide conjugate for thermally targeted delivery to solid tumors. Journal of controlled release : official journal of the Controlled Release Society 2006; 110(2): 362-9.
[53] Bidwell GL, 3rd, Davis AN, Fokt I, Priebe W, Raucher D. A thermally targeted elastin-like polypeptide-doxorubicin conjugate overcomes drug resistance. Investigational new drugs 2007; 25(4): 313-26.

[54] MacKay JA, Chen M, McDaniel JR, Liu W, Simnick AJ, Chilkoti A. Self-assembling chimeric polypeptidedoxorubicin conjugate nanoparticles that abolish tumours after a single injection. Nature materials 2009; 8(12): 99399.

[55] McDaniel JR, Bhattacharyya J, Vargo KB, Hassouneh W, Hammer DA, Chilkoti A. Self-assembly of thermally responsive nanoparticles of a genetically encoded peptide polymer by drug conjugation. Angewandte Chemie 2013; 52(6): 1683-7.

[56] George EM, Liu H, Robinson GG, Bidwell GL. A polypeptide drug carrier for maternal delivery and prevention of fetal exposure. Journal of drug targeting 2014; 22(10): 935-47.

[57] Rodríguez-Cabello JC, Martín L, Alonso M, Arias FJ, Testera AM. "Recombinamers" as advanced materials for the post-oil age. Polymer 2009; 50(22): 5159-69.

[58] Wright ER, Conticello VP. Self-assembly of block copolymers derived from elastin-mimetic polypeptide sequences. Adv Drug Deliv Rev 2002; 54(8): 1057-73.

[59] Martín L, Castro E, Ribeiro A, Alonso M, RodríguezCabello JC. Temperature-Triggered Self-Assembly of Elastin-Like Block Co-Recombinamers:The Controlled Formation of Micelles and Vesicles in an Aqueous Medium. Biomacromolecules 2012; 13(2): 293-98.

[60] Pinedo-Martin G, Castro E, Martin L, Alonso M, Rodriguez-Cabello JC. Effect of surfactants on the selfassembly of a model elastin-like block corecombinamer: from micelles to an aqueous two-phase system. Langmuir : the ACS journal of surfaces and colloids 2014; 30(12): 343240.

[61] Pinedo-Martín G, Santos M, Testera AM, Alonso M, Rodríguez-Cabello JC. The effect of $\mathrm{NaCl}$ on the selfassembly of elastin-like block co-recombinamers: Tuning the size of micelles and vesicles. Polymer 2014; 55(21): 531421.

[62] Shi P, Aluri S, Lin YA, et al. Elastin-based protein polymer nanoparticles carrying drug at both corona and core suppress tumor growth in vivo. Journal of controlled release : official journal of the Controlled Release Society 2013; 171(3): 330-8.

[63] Na K, Jung J, Lee J, Hyun J. Thermoresponsive pore structure of biopolymer microspheres for a smart drug carrier. Langmuir : the ACS journal of surfaces and colloids 2010; 26(13): 11165-9.

[64] Cheng J, Park M, Lim D, Hyun J. Polypeptide microgel capsules as drug carriers. Macromol Res 2013; 21(11): 116366.

[65] Costa RR, Castro E, Arias FJ, Rodriguez-Cabello JC, Mano JF. Multifunctional compartmentalized capsules with a hierarchical organization from the nano to the macro scales. Biomacromolecules 2013; 14(7): 2403-10. 
18 Journal Name, 2014, Vol. 0, No. OPrincipal Author Last Name et al.

[66] Costa RR, Girotti A, Santos M, Arias FJ, Mano JF, Rodriguez-Cabello JC. Cellular uptake of multilayered capsules produced with natural and genetically engineered biomimetic macromolecules. Acta biomaterialia 2014; 10(6): 2653-62.

[67] Han W, MacEwan SR, Chilkoti A, Lopez GP. Bioinspired synthesis of hybrid silica nanoparticles templated from elastin-like polypeptide micelles. Nanoscale 2015; 7(28): 12038-44.

[68] Assal Y, Mizuguchi Y, Mie M, Kobatake E. Growth Factor Tethering to Protein Nanoparticles via Coiled-Coil Formation for Targeted Drug Delivery. Bioconjug Chem 2015.

[69] Ciofani G, Genchi GG, Guardia P, Mazzolai B, Mattoli V, Bandiera A. Recombinant human elastin-like magnetic microparticles for drug delivery and targeting. Macromolecular bioscience 2014; 14(5): 632-42.

[70] Chen TH, Bae Y, Furgeson DY. Intelligent biosynthetic nanobiomaterials (IBNs) for hyperthermic gene delivery. Pharmaceutical research 2008; 25(3): 683-91.

[71] Pina MJ, Alex SM, Arias FJ, et al. Elastin-like recombinamers with acquired functionalities for genedelivery applications. Journal of biomedical materials research Part A 2015.

[72] Prabhakar U, Maeda H, Jain RK, et al. Challenges and key considerations of the enhanced permeability and retention effect for nanomedicine drug delivery in oncology. Cancer Res 2013; 73(8): 2412-7.

[73] Falk MH, Issels RD. Hyperthermia in oncology. Int J Hyperthermia 2001; 17(1): 1-18.

[74] Needham D, Anyarambhatla G, Kong G, Dewhirst MW. A new temperature-sensitive liposome for use with mild hyperthermia: characterization and testing in a human tumor xenograft model. Cancer Res 2000; 60(5): 1197-201.

[75] Liu W, Dreher MR, Furgeson DY, et al. Tumor accumulation, degradation and pharmacokinetics of elastinlike polypeptides in nude mice. Journal of controlled release : official journal of the Controlled Release Society 2006; 116(2): $170-8$.

[76] Dreher MR, Liu W, Michelich CR, Dewhirst MW, Chilkoti A. Thermal cycling enhances the accumulation of a temperature-sensitive biopolymer in solid tumors. Cancer Res 2007; 67(9): 4418-24.

[77] Wang F, Wang Y, Zhang X, Zhang W, Guo S, Jin F. Recent progress of cell-penetrating peptides as new carriers for intracellular cargo delivery. Journal of Controlled Release 2014; 174: 126-36.

[78] Kosuge M, Takeuchi T, Nakase I, Jones AT, Futaki S. Cellular Internalization and Distribution of Arginine-Rich Peptides as a Function of Extracellular Peptide Concentration, Serum, and Plasma Membrane Associated Proteoglycans. Bioconjugate Chemistry 2008; 19(3): 656-64.

[79] Thoren PE, Persson D, Isakson P, Goksor M, Onfelt A, Norden B. Uptake of analogs of penetratin, Tat(48-60) and oligoarginine in live cells. Biochem Biophys Res Commun 2003; 307(1): 100-7.
[80] Bidwell III GL, Davis AN, Raucher D. Targeting a cMyc inhibitory polypeptide to specific intracellular compartments using cell penetrating peptides. Journal of Controlled Release 2009; 135(1): 2-10.

[81] Bidwell III GL, Perkins E, Raucher D. A thermally targeted c-Myc inhibitory polypeptide inhibits breast tumor growth. Cancer Letters 2012; 319(2): 136-43.

[82] Bidwell GL, 3rd, Perkins E, Hughes J, Khan M, James JR, Raucher D. Thermally targeted delivery of a c-Myc inhibitory polypeptide inhibits tumor progression and extends survival in a rat glioma model. PloS one 2013; 8(1): e55104.

[83] Massodi I, Moktan S, Rawat A, Bidwell GL, 3rd, Raucher D. Inhibition of ovarian cancer cell proliferation by a cell cycle inhibitory peptide fused to a thermally responsive polypeptide carrier. Int J Cancer 2010; 126(2): 533-44.

[84] Bidwell III GL, Whittom AA, Thomas E, Lyons D, Hebert MD, Raucher D. A thermally targeted peptide inhibitor of symmetrical dimethylation inhibits cancer-cell proliferation. Peptides 2010; 31(5): 834-41.

[85] Bidwell GL, 3rd, Fokt I, Priebe W, Raucher D. Development of elastin-like polypeptide for thermally targeted delivery of doxorubicin. Biochem Pharmacol 2007; 73(5): 620-31.

[86] Moktan S, Ryppa C, Kratz F, Raucher D. A thermally responsive biopolymer conjugated to an acid-sensitive derivative of paclitaxel stabilizes microtubules, arrests cell cycle, and induces apoptosis. Investigational new drugs 2012; 30(1): 236-48.

[87] MacEwan SR, Chilkoti A. Applications of elastin-like polypeptides in drug delivery. Journal of controlled release : official journal of the Controlled Release Society 2014; 190: 314-30.

[88] Simnick AJ, Valencia CA, Liu R, Chilkoti A. Morphing low-affinity ligands into high-avidity nanoparticles by thermally triggered self-assembly of a genetically encoded polymer. ACS Nano 2010; 4(4): $2217-$ 27.

[89] Dreher MR, Simnick AJ, Fischer K, et al. Temperature triggered self-assembly of polypeptides into multivalent spherical micelles. J Am Chem Soc 2008; 130(2): 687-94.

[90] Hassouneh W, Fischer K, MacEwan SR, et al. Unexpected multivalent display of proteins by temperature triggered self-assembly of elastin-like polypeptide block copolymers. Biomacromolecules 2012; 13(5): 1598-605.

[91] MacEwan SR, Chilkoti A. Controlled apoptosis by a thermally toggled nanoscale amplifier of cellular uptake. Nano Lett 2014; 14(4): 2058-64.

[92] van Eldijk MB, Smits FC, Vermue N, Debets MF, Schoffelen S, van Hest JC. Synthesis and self-assembly of well-defined elastin-like polypeptide-poly(ethylene glycol) conjugates. Biomacromolecules 2014; 15(7): 2751-9.

[93] Balamuralidhara V, Pramodkumar T, Srujana N, et al. pH sensitive drug delivery systems: A review. 2011. 
[94] Måseide K, Kalliomäki T, Hill RP. Microenvironmental effects on tumour progression and metastasis. Integration/Interaction of Oncologic Growth: Springer; 2005. p. 1-22.

[95] Cardone RA, Casavola V, Reshkin SJ. The role of disturbed $\mathrm{pH}$ dynamics and the $\mathrm{Na}+\mathrm{H}+$ exchanger in metastasis. Nature Reviews Cancer 2005; 5(10): 786-95.

[96] MacKay JA, Callahan DJ, FitzGerald KN, Chilkoti A. Quantitative model of the phase behavior of recombinant $\mathrm{pH}$-responsive elastin-like polypeptides. Biomacromolecules 2010; 11(11): 2873-79.

[97] Koutsioumpa M, Papadimitriou E. Cell surface nucleolin as a target for anti-cancer therapies. Recent patents on anti-cancer drug discovery 2014; 9(2): 137-52.

[98] Porkka K, Laakkonen P, Hoffman JA, Bernasconi M, Ruoslahti E. A fragment of the HMGN2 protein homes to the nuclei of tumor cells and tumor endothelial cells in vivo. Proceedings of the National Academy of Sciences 2002; 99(11): 7444-49.

[99] Betre H, Liu W, Zalutsky MR, Chilkoti A, Kraus VB, Setton LA. A thermally responsive biopolymer for intraarticular drug delivery. Journal of Controlled Release 2006; 115(2): 175-82.

[100] Kimmerling KA, Furman BD, Mangiapani DS, et al. Sustained intra-articular delivery of IL-1RA from a thermally-responsive elastin-like polypeptide as a therapy for post-traumatic arthritis. Eur Cell Mater 2015; 29: 124-39; discussion 39-40.

[101] Bessa PC, Machado R, Nurnberger S, et al. Thermoresponsive self-assembled elastin-based nanoparticles for delivery of BMPs. Journal of controlled release : official journal of the Controlled Release Society 2010; 142(3): 312-8.

[102] Shamji MF, Whitlatch L, Friedman AH, Richardson WJ, Chilkoti A, Setton LA. An injectable and in situ-gelling biopolymer for sustained drug release following perineural administration. Spine (Phila Pa 1976) 2008; 33(7): 748-54.

[103] Sinclair SM, Bhattacharyya J, McDaniel JR, et al. A genetically engineered thermally responsive sustained release curcumin depot to treat neuroinflammation. Journal of controlled release : official journal of the Controlled Release Society 2013; 171(1): 38-47.

[104] Liu W, MacKay JA, Dreher MR, et al. Injectable intratumoral depot of thermally responsive polypeptideradionuclide conjugates delays tumor progression in a mouse model. Journal of controlled release : official journal of the Controlled Release Society 2010; 144(1): 2-9.

[105] Liu W, McDaniel J, Li X, et al. Brachytherapy using injectable seeds that are self-assembled from genetically encoded polypeptides in situ. Cancer Res 2012; 72(22): 5956-65.

[106] Amiram M, Luginbuhl KM, Li X, Feinglos MN, Chilkoti A. A depot-forming glucagon-like peptide-1 fusion protein reduces blood glucose for five days with a single injection. Journal of controlled release : official journal of the Controlled Release Society 2013; 172(1): 144-51.
[107] Nelson CM, Tien J. Microstructured extracellular matrices in tissue engineering and development. Current opinion in biotechnology 2006; 17(5): 518-23.

[108] Martin L, Alonso M, Moller M, Rodriguez-Cabello JC, Mela P. 3D microstructuring of smart bioactive hydrogels based on recombinant elastin-like polymers. Soft Matter 2009; 5(8): 1591-93.

[109] Falconnet D, Csucs G, Grandin HM, Textor M. Surface engineering approaches to micropattern surfaces for cell-based assays. Biomaterials 2006; 27(16): 3044-63.

[110] Numata K, Kaplan DL. Silk-based delivery systems of bioactive molecules. Adv Drug Deliv Rev 2010; 62(15): 1497-508.

[111] Fernandez-Colino A, Arias FJ, Alonso M, RodriguezCabello JC. Self-organized ECM-mimetic model based on an amphiphilic multiblock silk-elastin-like corecombinamer with a concomitant dual physical gelation process. Biomacromolecules 2014; 15(10): 3781-93.

[112] Gustafson J, Greish K, Frandsen J, Cappello J, Ghandehari H. Silk-elastinlike recombinant polymers for gene therapy of head and neck cancer: from molecular definition to controlled gene expression. Journal of Controlled Release 2009; 140(3): 256-61.

[113] Megeed Z, Haider M, Li D, O'Malley BW, Jr., Cappello J, Ghandehari H. In vitro and in vivo evaluation of recombinant silk-elastinlike hydrogels for cancer gene therapy. Journal of controlled release : official journal of the Controlled Release Society 2004; 94(2-3): 433-45.

[114] Megeed Z, Cappello J, Ghandehari H. Controlled release of plasmid DNA from a genetically engineered silkelastinlike hydrogel. Pharmaceutical research 2002; 19(7): 954-9.

[115] Greish K, Frandsen J, Scharff S, et al. Silk - elastinlike protein polymers improve the efficacy of adenovirus thymidine kinase enzyme prodrug therapy of head and neck tumors. The journal of gene medicine 2010; 12(7): 572-79.

[116] Gustafson JA, Price RA, Frandsen J, Henak CR, Cappello J, Ghandehari H. Synthesis and characterization of a matrix-metalloproteinase responsive silk-elastinlike protein polymer. Biomacromolecules 2013; 14(3): 618-25.

[117] Price R, Poursaid A, Cappello J, Ghandehari H. In vivo evaluation of matrix metalloproteinase responsive silkelastinlike protein polymers for cancer gene therapy. Journal of Controlled Release 2015; 213: 96-102.

[118] Amruthwar SS, Puckett AD, Janorkar AV. Preparation and characterization of novel elastin-like polypeptide - collagen composites. Journal of Biomedical Materials Research Part A 2013; 101(8): 2383-91.

[119] Amruthwar SS, Janorkar AV. Preparation and characterization of elastin-like polypeptide scaffolds for local delivery of antibiotics and proteins. J Mater Sci Mater Med 2012; 23(12): 2903-12.

[120] Lee J, Macosko CW, Urry DW. Mechanical properties of cross-linked synthetic elastomeric polypentapeptides. Macromolecules 2001; 34(17): 5968-74. 
[121] Nagapudi K, Brinkman WT, Leisen JE, et al. Photomediated solid-state cross-linking of an elastinmimetic recombinant protein polymer. Macromolecules 2002; 35(5): 1730-37.

[122] Lim DW, Nettles DL, Setton LA, Chilkoti A. Rapid cross-linking of elastin-like polypeptides with (hydroxymethyl) phosphines in aqueous solution. Biomacromolecules 2007; 8(5): 1463-70.

[123] McHale MK, Setton LA, Chilkoti A. Synthesis and in vitro evaluation of enzymatically cross-linked elastin-like polypeptide gels for cartilaginous tissue repair. Tissue engineering 2005; 11(11-12): 1768-79.

[124] González de Torre I, Quintanilla L, Pinedo-Martín G, Alonso M, Rodríguez-Cabello JC. Nanogel Formation from Dilute Solutions of Clickable Elastin-like Recombinamers and its Dependence on Temperature: Two Fractal Gelation Modes. ACS applied materials \& interfaces 2014; 6(16): 14509-15.

[125] de Torre IG, Wolf F, Santos M, et al. Elastin-like recombinamer-covered stents: Towards a fully biocompatible and non-thrombogenic device for cardiovascular diseases. Acta biomaterialia 2015; 12: 14655 .

[126] Mecham RP, Broekelmann TJ, Fliszar CJ, Shapiro SD, Welgus HG, Senior RM. Elastin degradation by matrix metalloproteinases Cleavage site specificity and mechanisms of elastolysis. Journal of Biological Chemistry 1997; 272(29): 18071-76.

[127] Bandiera A, Markulin A, Corich L, Vita F, Borelli V. Stimuli-induced release of compounds from elastin biomimetic matrix. Biomacromolecules 2013; 15(1): 416-22.

[128] Shi P, Gustafson JA, MacKay JA. Genetically engineered nanocarriers for drug delivery. International journal of nanomedicine 2014; 9: 1617.

[129] Altman GH, Diaz F, Jakuba C, et al. Silk-based biomaterials. Biomaterials 2003; 24(3): 401-16.

[130] Foo CWP, Kaplan DL. Genetic engineering of fibrous proteins: spider dragline silk and collagen. Advanced drug delivery reviews 2002; 54(8): 1131-43.

[131] Numata K, Reagan MR, Goldstein RH, Rosenblatt M, Kaplan DL. Spider silk-based gene carriers for tumor cellspecific delivery. Bioconjug Chem 2011; 22(8): 1605-10.

[132] Numata K, Mieszawska - Czajkowska AJ, Kvenvold LA, Kaplan DL. Silk-Based Nanocomplexes with Tumor-Homing Peptides for Tumor-Specific Gene Delivery. Macromolecular bioscience 2012; 12(1): 75-82.

[133] Mwangi TK, Bowles RD, Tainter DM, Bell RD, Kaplan DL, Setton LA. Synthesis and characterization of silk fibroin microparticles for intra-articular drug delivery. Int $\mathbf{J}$ Pharm 2015; 485(1-2): 7-14.

[134] Bettini S, Bonfrate V, Syrgiannis Z, et al. Biocompatible Collagen Paramagnetic Scaffold For Controlled Drug Release. Biomacromolecules 2015.

[135] Mundargi RC, Babu VR, Rangaswamy V, Patel P, Aminabhavi TM. Nano/micro technologies for delivering macromolecular therapeutics using poly (D, L-lactide-coglycolide) and its derivatives. Journal of Controlled Release 2008; 125(3): 193-209.

[136] Parveen S, Sahoo SK. Long circulating chitosan/PEG blended PLGA nanoparticle for tumor drug delivery. European journal of pharmacology 2011; 670(2): 372-83.

[137] Niu C, Wang Z, Lu G, et al. Doxorubicin loaded superparamagnetic PLGA-iron oxide multifunctional microbubbles for dual-mode US/MR imaging and therapy of metastasis in lymph nodes. Biomaterials 2013; 34(9): 2307 17.

[138] Schleich N, Sibret P, Danhier P, et al. Dual anticancer drug/superparamagnetic iron oxide-loaded PLGA-based nanoparticles for cancer therapy and magnetic resonance imaging. International journal of pharmaceutics 2013; 447(1): 94-101.

[139] Hutmacher D, Hürzeler MB, Schliephake H. A review of material properties of biodegradable and bioresorbable polymers and devices for GTR and GBR applications. The International journal of oral \& maxillofacial implants 1995; 11(5): 667-78.

[140] Turner CH, Takano Y, Owan I. Aging changes mechanical loading thresholds for bone formation in rats. Journal of Bone and Mineral Research 1995; 10(10): 154449.

[141] De la Riva B, Sánchez E, Hernández A, et al. Local controlled release of VEGF and PDGF from a combined brushite-chitosan system enhances bone regeneration. Journal of Controlled Release 2010; 143(1): 45-52.

[142] Porter RM, Huckle WR, Goldstein AS. Effect of dexamethasone withdrawal on osteoblastic differentiation of bone marrow stromal cells. Journal of cellular biochemistry 2003; 90(1): 13-22.

[143] Kim H, Suh H, Jo SA, et al. In vivo bone formation by human marrow stromal cells in biodegradable scaffolds that release dexamethasone and ascorbate-2-phosphate. Biochemical and biophysical research communications 2005; 332(4): 1053-60.

[144] Choi SH, Park TG. G-CSF loaded biodegradable PLGA nanoparticles prepared by a single oil-in-water emulsion method. International journal of pharmaceutics 2006; 311(1): 223-28.

[145] Bae SE, Son JS, Park K, Han DK. Fabrication of covered porous PLGA microspheres using hydrogen peroxide for controlled drug delivery and regenerative medicine. Journal of Controlled Release 2009; 133(1): 3743.

[146] Piao Z-G, Kim J-S, Son J-S, et al. Osteogenic evaluation of collagen membrane containing drug-loaded polymeric microparticles in a rat calvarial defect model. Tissue Engineering Part A 2014; 20(23-24): 3322-31.

[147] Nuttelman CR, Tripodi MC, Anseth KS. Dexamethasone - functionalized gels induce osteogenic differentiation of encapsulated hMSCs. Journal of Biomedical Materials Research Part A 2006; 76(1): 183-95. 
[148] Ganaha F, Kao EY, Wong H, et al. Stent-based controlled release of intravascular angiostatin to limit plaque progression and in-stent restenosis. Journal of vascular and interventional radiology 2004; 15(6): 601-08.

[149] Kaushal N, Durmaz YY, Bao L, Merajver SD, ElSayed ME. "Smart" Nanoparticles Enhance the Cytoplasmic Delivery of Anti-RhoC Silencing RNA and Inhibit the Migration and Invasion of Aggressive Breast Cancer Cells. Molecular pharmaceutics 2015.

[150] Kovochich M, Marsden MD, Zack JA. Activation of latent HIV using drug-loaded nanoparticles. PloS one 2011; 6(4): e18270.

[151] Beans EJ, Fournogerakis D, Gauntlett C, et al. Highly potent, synthetically accessible prostratin analogs induce latent HIV expression in vitro and ex vivo. Proceedings of the National Academy of Sciences 2013; 110(29): 11698703.

[152] Bear HD, McFadden AW, Kostuchenko PJ, et al. Bryostatin 1 activates splenic lymphocytes and induces sustained depletion of splenocyte protein kinase $\mathrm{C}$ activity in vivo after a single intravenous administration. Anti-cancer drugs 1996; 7(3): 299-306.

[153] Franzen S, Lommel SA. Targeting cancer with'smart bombs': equipping plant virus nanoparticles for a'seek and destroy'mission. Nanomedicine 2009; 4(5): 575-88.

[154] Ren Y, Wong SM, Lim LY. Application of plant viruses as nano drug delivery systems. Pharmaceutical research 2010; 27(11): 2509-13.

[155] Steinmetz NF, Manchester M. Viral nanoparticles: tools for material science and biomedicine: Pan Stanford Publishing; 2011.

[156] Esfandiari N, Arzanani MK, Soleimani M, KohiHabibi M, Svendsen WE. A new application of plant virus nanoparticles as drug delivery in breast cancer. Tumor Biology 2015: 1-8.

[157] Rieter WJ, Taylor KM, An H, Lin W, Lin W. Nanoscale metal-organic frameworks as potential multimodal contrast enhancing agents. Journal of the American Chemical Society 2006; 128(28): 9024-25.

[158] Hayashi H, Cote AP, Furukawa H, O'Keeffe M, Yaghi OM. Zeolite A imidazolate frameworks. Nature materials 2007; 6(7): 501-06.

[159] Adhikari C, Das A, Chakraborty A. Zeolitic imidazole framework (ZIF) nanospheres for easy encapsulation and controlled release of an anticancer drug doxorubicin under different external stimuli: A way towards smart drug delivery system. Molecular pharmaceutics 2015. 